\title{
Photochemical Approach to New Polycyclic Substrates Suitable for Further Photocatalytic Functionalization ${ }^{\dagger}$
}

\author{
Dragana Vuk, ${ }^{a}$ Željko Marinić, ${ }^{b}$ and Irena Škorića,* \\ ${ }^{a}$ Department of Organic Chemistry, Faculty of Chemical Engineering and Technology, \\ University of Zagreb, Marulićev trg 19, 10000 Zagreb, Croatia \\ ${ }^{\mathrm{b}}$ Center for NMR, Rudjer Bošković Institute, Bijenička cesta 54, 10000 Zagreb, Croatia
}

RECEIVED APRIL 3, 2014; REVISED OCTOBER 9, 2014; ACCEPTED NOVEMBER 6, 2014

\begin{abstract}
New polycyclic compounds are synthesized by photocycloaddition reactions of methoxy, methyl or phenyl substituted butadiene derivatives 11-14. Mono- and dimethoxy butadiene derivatives 11 and 12 undergo intramolecular [2+2] photocycloaddition giving benzobicyclo[3.2.1]octadiene structures (endo15, endo,trans-17) as main products. As minor photoproducts tricyclic compounds endo-16 and endo,trans-18 are isolated, respectively, formed by [4+2] photoinduced cycloaddition of the starting molecules. The reaction of compounds $\mathbf{1 3}$ and $\mathbf{1 4}$ is more selective and only benzobicyclo[3.2.1]octadienes endo,endo-19 and endo,endo-20 are formed, respectively. New bicyclo[3.2.1]octadienes with isolated double bond can be suitable substrates for further efficient photocatalytic oxygenations in course of new functionalized polycycles, potentially new biologically active compounds.
\end{abstract}

Keywords: cycloaddition, photochemistry, butadienes, benzobicyclo[3.2.1.]octadiene, polycycles

\section{INTRODUCTION}

Photochemistry of mono- and dibutadienyl derivatives 1a-2d (Figure 1) has been studied in detail. ${ }^{1-3}$ Unsubstituted starting compounds 1a and 2a, upon irradiation at $350 \mathrm{~nm}$, give as the main products of intramolecular cycloaddition benzobicyclo[3.2.1] octadiene structures endo-3 and endo,trans-4, respectively. ${ }^{1,2}$ The bicyclo[3.2.1]octane skeleton, saturated analogue of the bicyclo[3.2.1] octadiene, is found in numerous biologically important active natural products. ${ }^{4-20}$ Moreover, benzobicyclo[3.2.1]octadiene skeleton is also an important fragment in biologically active compounds or it can be used as a suitable substrate for further transfor-

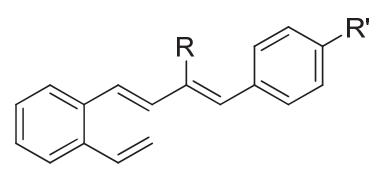

$$
\begin{aligned}
\text { 1a: } R=H, \quad R^{\prime}=H \\
\text { b: } R=\mathrm{CH}_{3}, \mathrm{R}^{\prime}=\mathrm{H} \\
\text { c: } \mathrm{R}=\mathrm{Cl}, \quad \mathrm{R}^{\prime}=\mathrm{H} \\
\text { d: } \mathrm{R}=\mathrm{H}, \quad \mathrm{R}^{\prime}=\mathrm{Cl}
\end{aligned}
$$

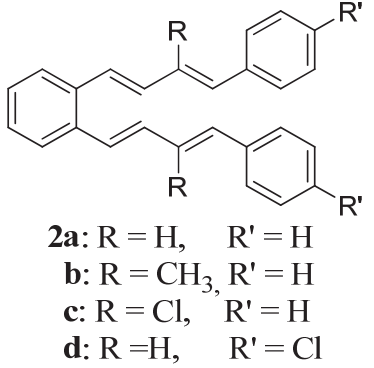

Figure 1. Structures of the known butadiene derivatives. mations on the isolated double bond, easily derivatized to new compounds with various functionalities. ${ }^{21-27}$

As a part of our increasing interest in the photochemistry of conjugated butadiene systems and to explore the effect of substituents on the butadiene units on the photochemical reactions, studies are expanded to methyl- and chloro-butadiene derivatives $\mathbf{1 b}-\mathbf{d}$ and $\mathbf{2 b}-\mathbf{d}^{2,3}$ Methyl group effects sterically to photochemical reactions, causing diverse photoinduced behaviour and formation of completely different photoproducts. ${ }^{2}$ Monosubstituted methyl derivative 1b upon irradiation gives dihydronaphtalene derivative 5 (Figure 2), while disubstituted methyl derivative $\mathbf{2 b}$ shows only geometric isomerization. Introduction of the chlorine atom to butadiene unit can lead the reaction course to various direction, under its steric and electronic effects. Upon irradiation, mono- and di- $\alpha$-chloro derivatives $\mathbf{1 c}$ and $\mathbf{2 c}$ photocyclize to give six-membered ring products $\mathbf{6}$ and 7. ${ }^{3}$ In continuation of our studies on photochemical behaviour of butadiene derivatives we extended research to $p$-substituted chloro derivatives 1d and 2d. ${ }^{3}$ The $p$ substitution increases molecular planarity, relative to $\alpha$ substitution and shift conformer equilibrium, affecting the reaction pathways and yields. As the main products upon irradiation of $p$-chloro-butadiene derivatives $\mathbf{1 d}$ and $\mathbf{2 d}$ new benzobicyclo[3.2.1]octa-diene structures endo-8 and

$\dagger$ Dedicated to Dr. Mirjana Eckert-Maksić on the occasion of her $70^{\text {th }}$ birthday.

* Author to whom correspondence should be addressed. (E-mail: irena.skoric@fkit.hr) 


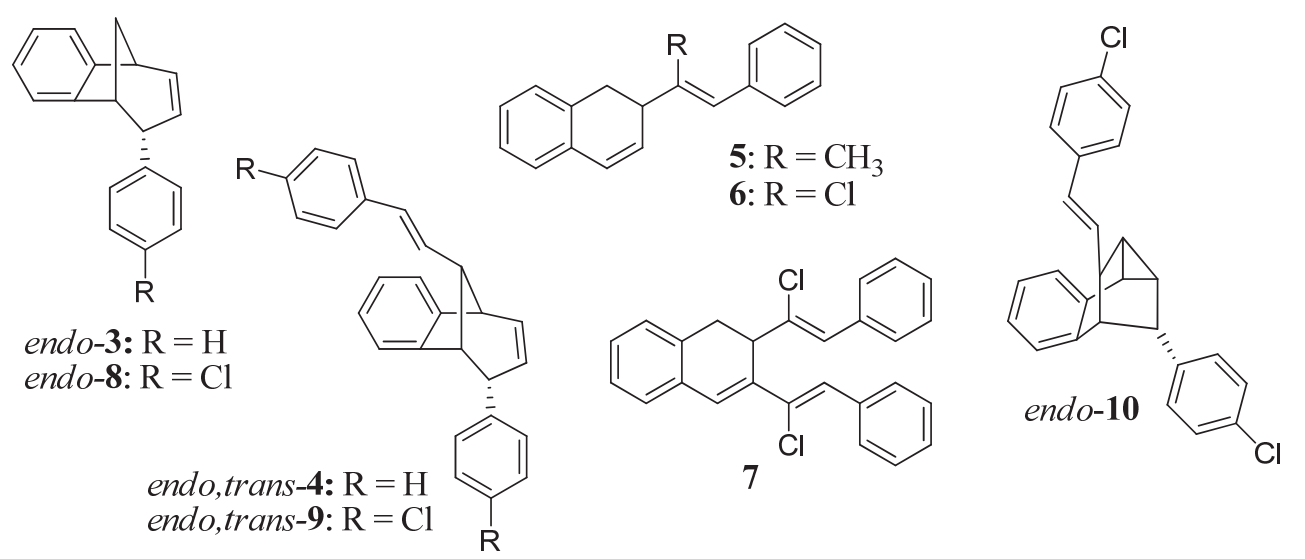

Figure 2. Structures of the isolated photoproducts.

endo,trans-9 are formed in very good yields, with smaller amounts $(10 \%)$ of dihydronaphtalene derivative endo-10 in case of $\mathbf{2 d}$ (Figure 2).

Starting compounds 1a-2d (Figure 1) possess very similar structures. Under steric and electronic effect of substituents, different products are formed. Therefore, understanding the influence and nature of substituents in the molecule is crucial for understanding the preffered reaction path and for prediction the behaviour of similar unresearched related compounds.

In the continuation of our research on photochemical behaviour of butadiene derivatives, we inserted one or two methoxy groups (as strong electron donating groups) to the $p$-position(s) of the aromatic $\operatorname{ring}(\mathrm{s})$ of the investigated compounds 11 and 12 (Figure 3). To explore the effect of methyl or phenyl group in the $\beta$ position of the $o$-vinyl group of 1 a (Figure 1) on photochemical behaviour, butadiene derivatives 13 and 14 (Figure 3) are prepared as related compounds to the molecule of 1a (Figure 1), also in course of the investigation the effect of substitution.

\section{EXPERIMENTAL}

\section{General Experimental Information}

The ${ }^{1} \mathrm{H}$ spectra were recorded on a spectrometer at 600 $\mathrm{MHz}$. The ${ }^{13} \mathrm{C}$ NMR spectra were registered at 150 $\mathrm{MHz}$, respectively. All NMR spectra were measured in $\mathrm{CDCl}_{3}$ using tetramethylsilane as reference. The assignment of the signals is based on 2D-CH correlation and 2D-HH-COSY experiments. UV spectra were measured on a UV/VIS Cary 50 spectrophotometer. IR spectra were recorded on a FTIR-ATR (film). Irradiation experiments were preformed in a Quartz vessel in toluene solution in a photochemical reactor equipped with $3500 \AA$ lamps. All irradiation experiments were carried out in deaerated solutions by bubbling a stream of argon prior to irradiation. Melting points were ob- tained using microscope equipped apparatus and are uncorrected. HRMS analysis were carried out on a mass spectrometer (MALDI TOF/TOF analyzer) equipped with $\mathrm{Nd}$ :YAG laser operating at $355 \mathrm{~nm}$ with firing rate $200 \mathrm{~Hz}$ in the positive $(\mathrm{H}+)$ or negative $(\mathrm{H}-)$ ion reflector mode. Silica gel $(0.063-0.2 \mathrm{~mm})$ was used for chromatographic purifications. Thin-layer chromatography (TLC) was performed silica gel $60 \mathrm{~F}_{254}$ plates. Solvents were purified by distillation.

Benzaldehyde and $p$-methoxy-cinnamaldehyde were obtained from a commercial source, $\beta, \beta-o$ xylyl(ditriphenylphosphonium) dibromide was prepared from $o$-xylyldibromide and triphenylphosphine in dimethylformamide.

The starting compounds 1-( $p$-methoxyphenyl)-4-(ostyryl)-1,3-butadiene (11), 1-\{o-[-4-( $p$-methoxyphe-nyl)1,3-butadienyl]phenyl $\}$-4-( $p$-methoxyphenyl)-1,3butadiene (12) and (1E,3E)-1-\{o-[(E)-1-propenyl]phenyl $\}$-4-phenyl-1,3-butadiene (trans,trans-13) are described. ${ }^{3}$ The data of the new compounds are given below.

(1Z,3E)-1-[(Z)-2-Stilbenyl]-4-phenyl-1,3-butadiene (cis, cis-14)

Starting compound $\mathbf{1 4}$ was prepared by Wittig reaction from $o$-xylylbis(triphenylphosphonium bromide) and
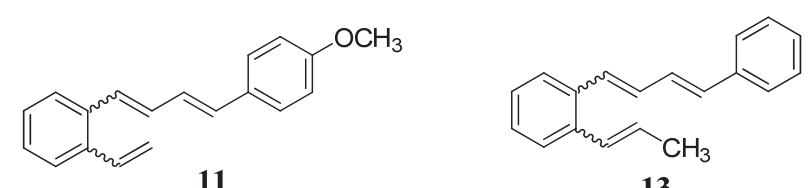

13<smiles>COc1ccc(/C=C/C=C/c2ccccc2/C=C/C=C/c2ccc(OC)cc2)cc1</smiles><smiles>C(=C/c1ccccc1/C=C/c1ccccc1)\c1ccccc1</smiles>

Figure 3. Structures of the starting butadiene derivatives 11-14. 

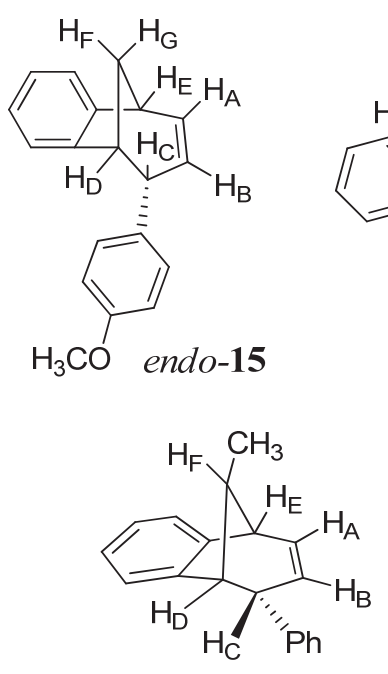

endo,endo-19
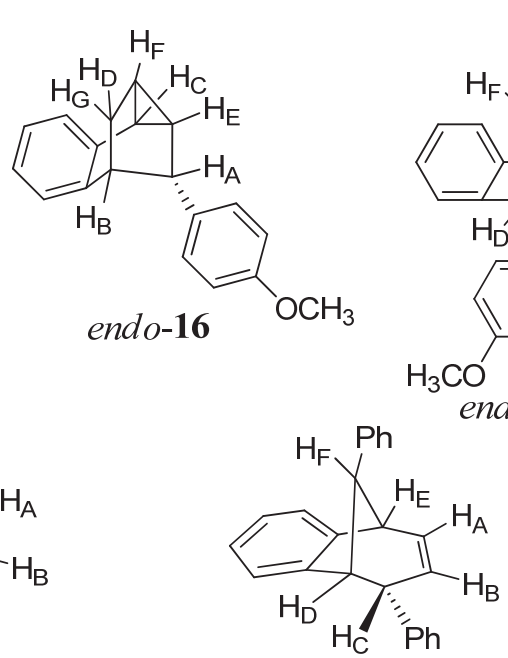

endo,endo-20

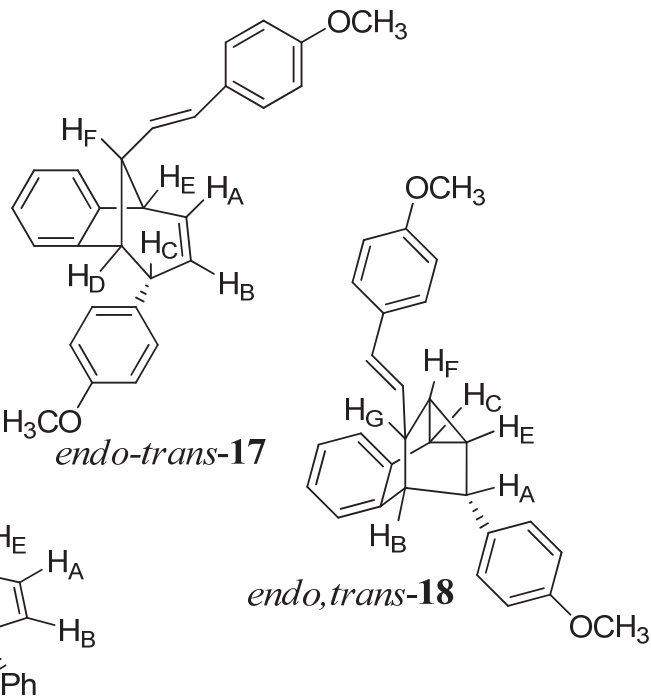

Chart 1. cinnamaldehyde. To a stirred solution of the triphenylphosphonium salt $(0.001 \mathrm{~mol})$ and the cinnamaldehyde $(0.011 \mathrm{~mol})$ in absolute ethanol $(200 \mathrm{~mL})$, a solution of sodium ethoxide $(0.253 \mathrm{~g}, 0.011 \mathrm{~mol}$ in 15 $\mathrm{mL}$ of absolute ethanol) was added dropwise. Stirring was continued under a stream of nitrogen for one hour at RT, when was 1.1 eq of benzaldehyde $(0.011 \mathrm{~mol})$ introduced and the next quantity of sodium ethoxide $(0.253 \mathrm{~g}, 0.011 \mathrm{~mol}$ in $15 \mathrm{~mL}$ of absolute ethanol) was added dropwise. The reaction was completed within 3-4 $\mathrm{h}$ (usually was left to stand overnight). After removal of the solvent, the residue was worked up with water and toluene. The toluene extracts were dried (anhydrous $\mathrm{MgSO}_{4}$ ), concentrated and the crude reaction mixture was purified. The reaction mixture contained cis,cis-, cis,trans-, trans, cis- and trans, trans-isomers in the ratio $3: 2: 2: 3$. After repeated column chromatography on silica gel using petroleum ether as the eluent only the cis,cis-14 was isolated and completely characterized.

cis,cis-14: yield $30 \%$; $R_{\mathrm{f}} \quad 0.88$ (petroleum ether/dichloromethane $=1: 1)$; colourless oil; UV $(96 \%$ EtOH) $\lambda_{\max } / \mathrm{nm}: 284$ and $254\left(\log \varepsilon / \mathrm{dm}^{3} \mathrm{~mol}^{-1} \mathrm{~cm}^{-1}\right.$ : $3.78(\mathrm{sh})$ and 3.93 ); IR $\tilde{v}_{\text {max. }} / \mathrm{cm}^{-1}$ (evaporated from $\mathrm{CH}_{2} \mathrm{Cl}_{2}$ ): 3016, 2923, 1492, 1446, 1218, 958, 774, 692; ${ }^{1} \mathrm{H}$ NMR $\left(\mathrm{CDCl}_{3} ; 300 \mathrm{MHz}\right) \delta / \mathrm{ppm}: 7.41(\mathrm{~d}, 1 \mathrm{H}, J=7.6$ $\mathrm{Hz}), 7.33$ (d, 2H, $J=7.5 \mathrm{~Hz}), 7.24-7.29$ (m, 4H), 7.20 $(\mathrm{t}, 1 \mathrm{H}, J=7.6 \mathrm{~Hz}), 7.07-7.18(\mathrm{~m}, 7 \mathrm{H}), 6.65(\mathrm{~d}, 1 \mathrm{H}, J=$ $15.6 \mathrm{~Hz}), 6.63(\mathrm{~d}, 1 \mathrm{H}, J=12.2 \mathrm{~Hz}), 6.61(\mathrm{~d}, 1 \mathrm{H}, J=$ $12.2 \mathrm{~Hz}), 6.56(\mathrm{~d}, 1 \mathrm{H}, J=11.3 \mathrm{~Hz}), 6.40(\mathrm{t}, 1 \mathrm{H}, J=$ 11.3); ${ }^{13} \mathrm{C}$ NMR $\left(\mathrm{CDCl}_{3} ; 75 \mathrm{MHz}\right) \delta / \mathrm{ppm}: 137.4$ (s), 136.9 (2s), 136.3 (s), 134.4 (d), 130.9 (d), 130.8 (d), 130.0 (d), 129.4 (d), 129.4 (d), 129.2 (d), 129.0 (2d), 128.6 (2d), 128.1 (2d), 127.6 (d), 127.1 (2d), 127.0 (d),
126.6 (2d), 125.5 (d); HRMS (TOF ES $\left.{ }^{+}\right) m / z$ for $\mathrm{C}_{24} \mathrm{H}_{20}$ : $\mathrm{M}^{+}$calcd $308.1559 ; \mathrm{M}^{+}$found 308.1559 .

\section{Irradiation Experiments}

A mixture of isomers of $\mathbf{1 1 - 1 4}$ in toluene $\left(3.0 \cdot 10^{-3} \mathrm{M}\right)$ was purged with argon for $20 \mathrm{~min}$ and irradiated at 350 $\mathrm{nm}$ in a Rayonet reactor (16 lamps) in a quartz vessel

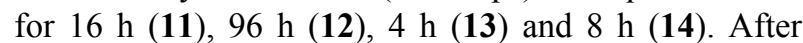
irradiation the solvent was removed in vacuo and the oily residue chromatographed on a silica gel column using petroleum ether as the eluent. The photoproducts endo-15, endo-16 (obtained from 11) 17, 18 (from 12), endo,endo-19 (from 13) and endo,endo-20 (from 14) (Chart 1) were isolated from the enriched first chromatographic fractions followed by a mixture of several unidentified products $(<2 \%)$. High-molecular-weight products remained on the column.

(11S)-11-(4-methoxyphenyl)tricyclo[6.3.1.0 ${ }^{2}, 7$ dodeca2,4,6,9-tetraene (endo-15):

Yield $55 \% ; R_{\mathrm{f}} 0.27$ (petroleum ether/dichloromethane = 8:2); colourless crystals; m.p. $45-47{ }^{\circ} \mathrm{C}$; UV (96\% EtOH) $\lambda_{\max } / \mathrm{nm}: 284,276,269$ and $226\left(\log \varepsilon / \mathrm{dm}^{3}\right.$ $\mathrm{mol}^{-1} \mathrm{~cm}^{-1}: 3.55,3.67,3.65$ and 4.16); IR $\tilde{v}_{\text {max. }} / \mathrm{cm}^{-1}$ (evaporated from $\left.\mathrm{CH}_{2} \mathrm{Cl}_{2}\right)$ : 2952, $1605(\mathrm{C}=\mathrm{C}$, ar), 1510 $(\mathrm{C}=\mathrm{C}), 1249\left(\mathrm{C}_{\mathrm{ar}}-\mathrm{O}-\mathrm{CH}_{3}\right), 1174,1035,771 ;{ }^{1} \mathrm{H}$ NMR $\left(\mathrm{CDCl}_{3}, 600 \mathrm{MHz}\right) \delta / \mathrm{ppm}: 7.11(\mathrm{~d}, 1 \mathrm{H}, J=7.3 \mathrm{~Hz})$, 7.03 (dt, 1H, $J=7.3 \mathrm{~Hz} ; 1.0 \mathrm{~Hz}), 6.82(\mathrm{dt}, 1 \mathrm{H}, J=7.3$ $\mathrm{Hz} ; 1.0 \mathrm{~Hz}), 6.72$ (d, 2H, $J=8.6 \mathrm{~Hz}), 6.64$ (d, 2H, $J=$ $8.6 \mathrm{~Hz}), 6.32-6.36\left(\mathrm{~m}, 1 \mathrm{H}, \mathrm{H}_{\mathrm{A}}\right), 6.25(\mathrm{~d}, 1 \mathrm{H}, J=7.3$ $\mathrm{Hz}), 5.28\left(\mathrm{dt}, 1 \mathrm{H}, \mathrm{H}_{\mathrm{B}}, J_{\mathrm{AB}}=9.6 \mathrm{~Hz} ; J_{\mathrm{BC}}=2.6 \mathrm{~Hz}\right)$, 3.91-3.94 (m, 1H, $\left.\mathrm{H}_{\mathrm{C}}\right), 3.77\left(\mathrm{~s}, 3 \mathrm{H},-\mathrm{OCH}_{3}\right), 3.35(\mathrm{t}$, $\left.1 \mathrm{H}, J_{\mathrm{DC}}=J_{\mathrm{DF}}=4.5 \mathrm{~Hz}, \mathrm{H}_{\mathrm{D}}\right), 3.27\left(\mathrm{dd}, 1 \mathrm{H}, J_{\mathrm{EA}}=6.3 \mathrm{~Hz}\right.$; 
$\left.J_{\mathrm{EF}}=4.7 \mathrm{~Hz}, \mathrm{H}_{\mathrm{E}}\right), 2.49-2.53\left(\mathrm{~m}, 1 \mathrm{H}, \mathrm{H}_{\mathrm{F}}\right), 2.37(\mathrm{~d}, 1 \mathrm{H}$, $\left.J_{\mathrm{GF}}=9.9 \mathrm{~Hz}, \mathrm{H}_{\mathrm{G}}\right) ;{ }^{13} \mathrm{C} \mathrm{NMR}\left(\mathrm{CDCl}_{3}, 75 \mathrm{MHz}\right) \delta / \mathrm{ppm}$ : 157.6 (s), 152.0 (s), 141.7 (s), 134.2 (s), 134.1 (d), 128.8 (2d), 126.3 (d), 125.8 (d), 125.5 (d), 124.5 (d), 119.6 (d), 112.6 (2d), 43.7 (t); HRMS (TOF $\mathrm{ES}^{+}$) $\mathrm{m} / \mathrm{z}$ for $\mathrm{C}_{19} \mathrm{H}_{18} \mathrm{O}: \mathrm{M}_{\text {calcd }}^{+} 262.1352 ; \mathrm{M}_{\text {found }}^{+} 262.1351$.

(10S)-10-(4-methoxyphenyl)tetracy-

clo[7.2.1. $\left.0^{2},{ }^{11} .0^{3},{ }^{8}\right]$ dodeca-3,5,7-triene (endo-16)

Yield $13 \% ; R_{\mathrm{f}} 0.21$ (petroleum ether/dichloromethane $=$ 8:2); colourless crystals; m.p. 91-93 ${ }^{\circ} \mathrm{C}$; UV (96 \% EtOH) $\lambda_{\max } / \mathrm{nm}: 285,277,270$ and $227\left(\log \varepsilon / \mathrm{dm}^{3}\right.$ $\mathrm{mol}^{-1} \mathrm{~cm}^{-1}: 4.10,3.27,3.19$ and 4.18); ${ }^{1} \mathrm{H}$ NMR $\left(\mathrm{CDCl}_{3}, 600 \mathrm{MHz}\right) \delta / \mathrm{ppm}: 7.26(\mathrm{~d}, 1 \mathrm{H}, J=7.4 \mathrm{~Hz})$, 7.06 (dt, 1H, $J=7.4 \mathrm{~Hz} ; 0.8 \mathrm{~Hz}), 6.89(\mathrm{dt}, 1 \mathrm{H}, J=7.4$ $\mathrm{Hz} ; 0.8 \mathrm{~Hz}), 6.79$ (d, 2H, $J=8.6 \mathrm{~Hz}), 6.66(\mathrm{~d}, 1 \mathrm{H}, J=$ $7.4 \mathrm{~Hz}), 6.55(\mathrm{~d}, 2 \mathrm{H}, J=8.6 \mathrm{~Hz}), 3.65\left(\mathrm{~s}, 3 \mathrm{H},-\mathrm{OCH}_{3}\right)$, $3.37\left(\mathrm{dd}, 1 \mathrm{H}, J_{\mathrm{AB}}=4.6 \mathrm{~Hz} ; J_{\mathrm{AE}}=2.8 \mathrm{~Hz}, \mathrm{H}_{\mathrm{A}}\right), 3.16(\mathrm{t}$, $\left.1 \mathrm{H}, J_{\mathrm{AB}}=J_{\mathrm{BD}}=4.6 \mathrm{~Hz}, \mathrm{H}_{\mathrm{B}}\right), 2.33\left(\mathrm{t}, 1 \mathrm{H}, J_{\mathrm{CE}}=J_{\mathrm{CF}}=7.2\right.$ $\mathrm{Hz}, \mathrm{H}_{\mathrm{C}}$ ), $2.12\left(\mathrm{ddd}, 1 \mathrm{H}, J_{\mathrm{DG}}=11.7 \mathrm{~Hz} ; J_{\mathrm{BD}}=4.6 \mathrm{~Hz}\right.$; $\left.J_{\mathrm{DF}}=2.8 \mathrm{~Hz}, \mathrm{H}_{\mathrm{D}}\right), 1.88\left(\mathrm{dt}, 1 \mathrm{H}, J_{\mathrm{CE}}=J_{\mathrm{EF}}=7.2 \mathrm{~Hz} ; J_{\mathrm{AE}}\right.$ $\left.=2.8 \mathrm{~Hz}, \mathrm{H}_{\mathrm{E}}\right), 1.77\left(\mathrm{dt}, 1 \mathrm{H}, J_{\mathrm{CE}}=J_{\mathrm{EF}}=7.2 \mathrm{~Hz} ; J_{\mathrm{AE}}=\right.$ $\left.2.8 \mathrm{~Hz}, \mathrm{H}_{\mathrm{F}}\right), 1.19\left(\mathrm{~d}, 1 \mathrm{H}, J_{\mathrm{DG}}=11.7 \mathrm{~Hz}, \mathrm{H}_{\mathrm{G}}\right) ;{ }^{13} \mathrm{C} \mathrm{NMR}$ $\left(\mathrm{CDCl}_{3}, 75 \mathrm{MHz}\right) \delta / \mathrm{ppm}: 157.0$ (s), 136.3 (s), 135.2 (s), 132.3 (s), 128.0 (2d), 125.3 (d), 124.9 (d), 124.5 (d), 123.8 (d), 112.5 (2d), 29.5 (t).

(11S)-11-(4-methoxyphenyl)-12-[(E)-2-(4-methoxyphenyl)ethenyl] tricyclo[6.3.1.0 $\left.{ }^{2}, 7\right]$ dodeca-2,4,6,9-tetraene (endo, trans-17)

Yield $24 \% ; R_{\mathrm{f}} 0.22$ (petroleum ether/dichloromethane $=$ 8:2); colourless oil; UV (96\% EtOH) $\lambda_{\max } / \mathrm{nm}$ : 260 and $229\left(\log \varepsilon / \mathrm{dm}^{3} \mathrm{~mol}^{-1} \mathrm{~cm}^{-1}: 4.28\right.$ and 4.22); IR $\tilde{v}_{\text {max. }} / \mathrm{cm}^{-1}$ (evaporated from $\left.\mathrm{CH}_{2} \mathrm{Cl}_{2}\right): 3021,1607(\mathrm{C}=\mathrm{C}$, ar), $1510(\mathrm{C}=\mathrm{C}), 1247\left(\mathrm{C}_{\mathrm{ar}}-\mathrm{O}-\mathrm{CH}_{3}\right), 1034,833,737 ;{ }^{1} \mathrm{H}$ NMR $\left(\mathrm{CDCl}_{3}, 600 \mathrm{MHz}\right) \delta / \mathrm{ppm}: 7.32$ (d, 2H, $J=8.5$ $\mathrm{Hz}), 7.14$ (d, 1H, $J=7.3 \mathrm{~Hz}), 7.07$ (dt, $1 \mathrm{H}, J=7.3 ; 0.9$ $\mathrm{Hz}), 6.95(\mathrm{~d}, 2 \mathrm{H}, J=8.5 \mathrm{~Hz}), 6.83-6.88(\mathrm{~m}, 2 \mathrm{H}), 6.72$ $(\mathrm{d}, 2 \mathrm{H}, J=8.7 \mathrm{~Hz}), 6.65(\mathrm{~d}, 2 \mathrm{H}, J=8.7 \mathrm{~Hz}), 6.41$ (ddd, $1 \mathrm{H}, J=8.3 ; 3.2 ; 2.5 \mathrm{~Hz}), 6.25-6.29(\mathrm{~m}, 2 \mathrm{H}), 5.32-5.33$ $(\mathrm{m}, 1 \mathrm{H}), 3.97-4.00\left(\mathrm{~m}, 2 \mathrm{H}, \mathrm{H}_{\mathrm{C}}\right), 3.85\left(\mathrm{~s}, 3 \mathrm{H},-\mathrm{OCH}_{3}\right)$, $3.77\left(\mathrm{~s}, 3 \mathrm{H},-\mathrm{OCH}_{3}\right), 3.75-3.79\left(\mathrm{~m}, 1 \mathrm{H}, \mathrm{H}_{\mathrm{D}}\right), 3.24(\mathrm{~d}$, $\left.1 \mathrm{H}, J=4.7 \mathrm{~Hz}, \mathrm{H}_{\mathrm{E}} / \mathrm{H}_{\mathrm{F}}\right), 3.20\left(\mathrm{~d}, 1 \mathrm{H}, J=6.6 \mathrm{~Hz}, \mathrm{H}_{\mathrm{E}} /\right.$ $\left.\mathrm{H}_{\mathrm{F}}\right) ;{ }^{13} \mathrm{C} \mathrm{NMR}\left(\mathrm{CDCl}_{3}, 75 \mathrm{MHz}\right) \delta / \mathrm{ppm}: 158.0(\mathrm{~s})$, 157.7 (s), 150.5 (s), 150.2 (s), 140.0 (s), 139.7 (s), 134.1 (d), 132.7 (d), 129.5 (2d), 128.9 (2d), 128.2 (d), 126.8 (d), 126.7 (d), 126.7 (d), 126.5 (d), 125.9 (d), 125.9 (d), 124.9 (d), 124.8 (d), 120.7 (d), 113.3 (q), 112.7 (q), 54.7 (d), 53.7 (d), 46.4 (d), 45.8 (d); HRMS (TOF ES ${ }^{+}$) $\mathrm{m} / \mathrm{z}$ for $\mathrm{C}_{28} \mathrm{H}_{26} \mathrm{O}_{2}: \quad[\mathrm{M}+\mathrm{K}]^{+}$calcd $433.1564 ; \quad[\mathrm{M}+\mathrm{K}]^{+}$found 433.1581 .

(10S)-10-(4-methoxyphenyl)-12-[(E)-2-(4-methoxyphenyl)ethenyl] tetracyclo $\quad\left[7.2 .1 .0^{2},{ }^{11} .0^{3},{ }^{8}\right]$ dodeca-3,5,7triene (endo, trans-18)

Yield $7 \% ; R_{\mathrm{f}} 0.20$ (petroleum ether/dichloromethane $=$ 8:2); colourless oil; UV (96 \% EtOH) $\lambda_{\max } / \mathrm{nm}: 274,264$ and $230\left(\log \varepsilon / \mathrm{dm}^{3} \mathrm{~mol}^{-1} \mathrm{~cm}^{-1}: 4.24(\mathrm{sh}), 4.33\right.$ and 4.14); IR $\tilde{v}_{\max } / \mathrm{cm}^{-1}$ (evaporated from $\mathrm{CH}_{2} \mathrm{Cl}_{2}$ ): 2922, $1606(\mathrm{C}=\mathrm{C}, \mathrm{ar}), 1510(\mathrm{C}=\mathrm{C}), 1247\left(\mathrm{C}_{\mathrm{ar}}-\mathrm{O}-\mathrm{CH}_{3}\right), 1076$, 1033, 964; ${ }^{1} \mathrm{H}$ NMR $\left(\mathrm{CDCl}_{3}, 600 \mathrm{MHz}\right) \delta / \mathrm{ppm}: 7.28(\mathrm{~d}$, $1 \mathrm{H}, J=7.6 \mathrm{~Hz}), 7.06(\mathrm{dt}, 1 \mathrm{H}, J=7.6 ; 1.0 \mathrm{~Hz}), 7.00(\mathrm{~d}$, $2 \mathrm{H}, J=8.7 \mathrm{~Hz}), 6.88(\mathrm{dt}, 1 \mathrm{H}, J=7.6 ; 1.0 \mathrm{~Hz}), 6.80(\mathrm{~d}$, $2 \mathrm{H}, J=8.7 \mathrm{~Hz}), 6.71(\mathrm{~d}, 2 \mathrm{H}, J=8.7 \mathrm{~Hz}), 6.61(\mathrm{~d}, 1 \mathrm{H}, J$ $=7.6 \mathrm{~Hz}), 6.56(\mathrm{~d}, 2 \mathrm{H}, J=8.7 \mathrm{~Hz}), 6.32(\mathrm{~d}, 1 \mathrm{H}, J=$ $15.7 \mathrm{~Hz}), 5.25$ (dd, $1 \mathrm{H}, J=15.7 ; 8.2 \mathrm{~Hz}), 3.73$ (s, 3H, $\left.\mathrm{OCH}_{3}\right), 3.66\left(\mathrm{~s}, 3 \mathrm{H},-\mathrm{OCH}_{3}\right), 3.51-3.54\left(\mathrm{~m}, 1 \mathrm{H}, \mathrm{H}_{\mathrm{A}}\right)$, $3.20\left(\mathrm{t}, 1 \mathrm{H}, J=4.6 \mathrm{~Hz}, \mathrm{H}_{\mathrm{B}}\right), 3.11-3.16\left(\mathrm{~m}, 1 \mathrm{H}, \mathrm{H}_{\mathrm{G}}\right)$, $2.40\left(\mathrm{t}, 1 \mathrm{H}, J=7.1 \mathrm{~Hz}, \mathrm{H}_{\mathrm{C}}\right), 1.95(\mathrm{dt}, 1 \mathrm{H}, J=6.4 ; 1.8$ $\left.\mathrm{Hz}, \mathrm{H}_{\mathrm{E}}\right), 1.81\left(\mathrm{dt}, 1 \mathrm{H}, J=6.4 ; 1.8 \mathrm{~Hz}, \mathrm{H}_{\mathrm{F}}\right) ;{ }^{13} \mathrm{C} \mathrm{NMR}$ $\left(\mathrm{CDCl}_{3}, 75 \mathrm{MHz}\right) \delta / \mathrm{ppm}$ : 158.4 (s), 157.6 (s), 136.4 (s), 133.3 (s), 132.2 (s), 130.6 (d), 129.8 (d), 129.0 (d), 128.6 (2d), 127.1 (d), 126.8 (d), 126.0 (d), 125.2 (d), 124.5 (d), 113.7 (d), 113.7 (d), 112.9 (2d), 55.3 (d), 55.0 (d), 48.3 (d), 43.8 (d), 38.8 (d), 22.7 (d), 21.5 (d), 17.9 (d); HRMS (TOF $\mathrm{ES}^{+}$) $\mathrm{m} / \mathrm{z}$ for $\mathrm{C}_{28} \mathrm{H}_{26} \mathrm{O}_{2}: \mathrm{M}^{+}$calcd 394.1927; $\mathrm{M}^{+}$found 394.1920.

(11S)-12-methyl-11-phenyltricyclo[6.3.1.02, $\left.{ }^{7}\right]$ dodeca2,4,6,9-tetraene (endo, endo-19)

Yield $77 \% ; R_{\mathrm{f}} 0.88$ (petroleum ether/dichloromethane $=$ 1:1); colourless oil; UV (96\% EtOH) $\lambda_{\max } / \mathrm{nm}: 324$, 275, 268 and $259\left(\log \varepsilon / \mathrm{dm}^{3} \mathrm{~mol}^{-1} \mathrm{~cm}^{-1}\right.$ : 2.94, 3.00, 3.02 and $2.96(\mathrm{sh})$ ); IR $\tilde{v}_{\max } / \mathrm{cm}^{-1}$ (evaporated from $\mathrm{CH}_{2} \mathrm{Cl}_{2}$ ): 3025, 1601, 1495, 1450, 1218, 1032, 755, 698; ${ }^{1} \mathrm{H} \mathrm{NMR}\left(\mathrm{CDCl}_{3}, 600 \mathrm{MHz}\right) \delta / \mathrm{ppm}: 7.14-7.18(\mathrm{~m}, 3 \mathrm{H})$, $7.10(\mathrm{~d}, 1 \mathrm{H}, J=7.4 \mathrm{~Hz}), 7.03(\mathrm{dt}, 1 \mathrm{H}, J=7.4 ; 0.8 \mathrm{~Hz})$, $6.80(\mathrm{dt}, 1 \mathrm{H}, J=7.4 ; 0.8, \mathrm{~Hz}), 6.70-6.74(\mathrm{~m}, 2 \mathrm{H}), 6.36-$ $6.42\left(\mathrm{~m}, 1 \mathrm{H}, \mathrm{H}_{\mathrm{A}}\right), 6.16(\mathrm{~d}, 1 \mathrm{H}, J=7.4 \mathrm{~Hz}), 5.30-5.32$ $\left(\mathrm{m}, 1 \mathrm{H}, \mathrm{H}_{\mathrm{B}}\right), 3.96-4.00\left(\mathrm{~m}, 1 \mathrm{H}, \mathrm{H}_{\mathrm{C}}\right), 3.04(\mathrm{~d}, 1 \mathrm{H}, J=$ $\left.4.8 \mathrm{~Hz}, \mathrm{H}_{\mathrm{D}}\right), 2.96\left(\mathrm{~d}, 1 \mathrm{H}, J=6.6 \mathrm{~Hz}, \mathrm{H}_{\mathrm{E}}\right), 2.75(\mathrm{dd}, 1 \mathrm{H}$, $\left.J=6.6,13.1 \mathrm{~Hz}, \mathrm{H}_{\mathrm{F}}\right), 0.93\left(\mathrm{~d}, 3 \mathrm{H}, J=6.6 \mathrm{~Hz},-\mathrm{CH}_{3}\right)$; ${ }^{13} \mathrm{C} \mathrm{NMR}\left(\mathrm{CDCl}_{3}, 150 \mathrm{MHz}\right) \delta / \mathrm{ppm}: 150.5$ (s), 141.9 (s), 139.8 (s), 135.1 (d), 127.9 (2d), 127.1 (2d), 126.9 (d), 125.8 (d), 125.7 (d), 125.6 (d), 124.6 (d), 120.9 (d), 55.0 (d), 49.1 (d), 47.1 (d), 46.7 (d), 17.8 (q); HRMS (TOF $\mathrm{ES}^{+}$) $m / z$ for $\mathrm{C}_{19} \mathrm{H}_{18}:[\mathrm{M}-\mathrm{H}]^{-}$calcd 245.1325; $[\mathrm{M}-\mathrm{H}]^{-}$found 245.1329 .

(11S)-11,12-diphenyltricyclo[6.3.1.0 $0^{2}, 7$ dodeca-2,4,6,9tetraene (endo,endo-20)

Yield $45 \% ; R_{\mathrm{f}} 0.88$ (petroleum ether/dichloromethane $=$ 1:1); UV (96\% EtOH) $\lambda_{\max } / \mathrm{nm}: 278$ and $253(\log \varepsilon /$ $\mathrm{dm}^{3} \mathrm{~mol}^{-1} \mathrm{~cm}^{-1}: 3.55$ (sh) and 3.71; IR $\tilde{v}_{\text {max }} / \mathrm{cm}^{-1}$ (evaporated from $\mathrm{CH}_{2} \mathrm{Cl}_{2}$ ): 3022, 2956, 1452, 1375, 1016, 746, 701; ${ }^{1} \mathrm{H}$ NMR $\left(\mathrm{CDCl}_{3}, 600 \mathrm{MHz}\right) \delta / \mathrm{ppm}: 7.16$ $7.20(\mathrm{~m}, 3 \mathrm{H}), 7.12$ (d, 2H, $J=7.6 \mathrm{~Hz}), 7.08$ (d, 2H, $J=$ $7.6 \mathrm{~Hz}), 7.03$ (d, 2H, $J=7.6 \mathrm{~Hz}), 7.00-7.03(\mathrm{~m}, 1 \mathrm{H})$, $6.80(\mathrm{dt}, 1 \mathrm{H}, J=7.7 ; 0.9 \mathrm{~Hz}), 6.75-6.78(\mathrm{~m}, 2 \mathrm{H}), 6.50$ $6.55\left(\mathrm{~m}, 1 \mathrm{H}, \mathrm{H}_{\mathrm{A}}\right), 6.15(\mathrm{~d}, 1 \mathrm{H}, J=7.3 \mathrm{~Hz}), 5.42$ (ddd, $\left.1 \mathrm{H}, J=9.7 ; 3.5 ; 1.7 \mathrm{~Hz}, \mathrm{H}_{\mathrm{B}}\right), 4.16-4.20\left(\mathrm{~m}, 1 \mathrm{H}, \mathrm{H}_{\mathrm{C}}\right)$, $3.88\left(\mathrm{~s}, 1 \mathrm{H}, \mathrm{H}_{\mathrm{F}}\right), 3.57\left(\mathrm{~d}, 1 \mathrm{H}, J=4.7 \mathrm{~Hz}, \mathrm{H}_{\mathrm{D}}\right), 3.48(\mathrm{~d}$, $\left.1 \mathrm{H}, J=6.7 \mathrm{~Hz}, \mathrm{H}_{\mathrm{E}}\right) ;{ }^{13} \mathrm{C} \mathrm{NMR}\left(\mathrm{CDCl}_{3}, 150 \mathrm{MHz}\right)$ 
<smiles>C=Cc1ccccc1/C=C/C=C/c1ccc(OC)cc1</smiles>

11<smiles>COc1ccc(/C=C/C=C/c2ccc(OC)cc2)cc1</smiles><smiles>C/C=C/c1ccccc1/C=C/C=C/c1ccccc1</smiles>

13<smiles>C(=C/c1ccccc1/C=C/c1ccccc1)\c1ccccc1</smiles>

14<smiles>COc1ccc(C2C=CC3CC2CC32Oc3ccccc32)cc1</smiles>

endo-15

hv $350 \mathrm{~nm}$ toluene

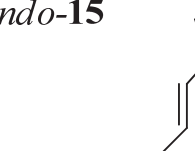<smiles>COC1CCCC1</smiles>
endo-16

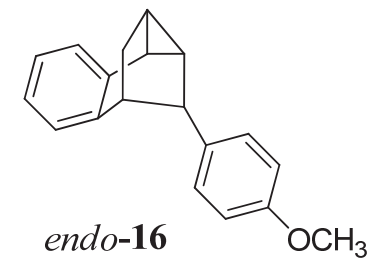

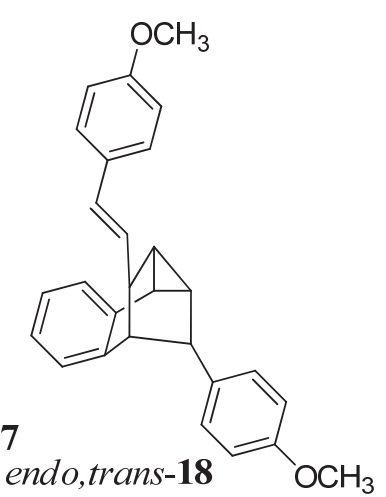<smiles>CC1C2C=CC(c3ccccc3)C(c3ccccc3)C1c1ccccc12</smiles>

endo,endo-19

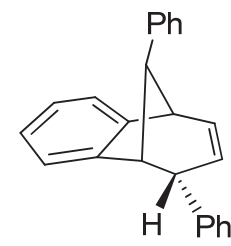

endo,endo-20

Scheme 1.

$\delta /$ ppm: 150.5 (s), 143.6 (s), 141.8 (s), 139.8 (s), 135.2 (d), 128.0 (2d), 127.6 (d), 127.3 (2d), 126.9 (d), 126.3 (d), 126.24 (d), 126.22 (d), 126.1 (2d), 125.9 (d), 125.5 (d), 124.9 (d), 120.4 (d), 58.8 (d), 54.2 (d), 47.6 (d), 46.6 (d). $\mathrm{HRMS}$ (TOF $\mathrm{ES}^{+}$) $\mathrm{m} / z$ for $\mathrm{C}_{24} \mathrm{H}_{20}$ : $[\mathrm{M}-\mathrm{H}]^{-}$calcd 307.1481; [M-H] ${ }^{-}$found 307.1493 .

\section{RESULTS AND DISCUSSION}

Starting compounds 11-14 are prepared by Wittig reaction according to the procedure described for butadienes in previous papers ${ }^{1-3}$ from $o$-xylylbis(triphenylphosphonium bromide) and corresponding aldehydes. The products are obtained as mixture of two (in case of 11), three (in case of 12) or four (in case of $\mathbf{1 3}$ and 14) isomers and subjected to irradiation. Irradiation experiments are performed in Rayonet reactor at $350 \mathrm{~nm}$ in toluene solutions under anaerobic conditions at low concentrations. Obtained photoproducts are isolated and completely characterized by spectroscopic methods.

Mono- and di-methoxy derivatives $\mathbf{1 1}$ and $\mathbf{1 2}$ undergo photochemical reaction to form new polycyclic structures 15-18 (Scheme 1). On irradiation of monomethoxy derivative 11, bicyclic derivative 15 was isolated as main product (55\%), with $13 \%$ of tricyclic derivative 16, while the high-molecular-weight products remained on the column (Scheme 1). The proposed mechanism of the formation of benzobicyclo[3.2.1] structure 15 involves intramolecular [2+2] cycloaddition via biradical intermediate $\mathbf{1 5}$ ' followed by preffered 1,6 ring closure. Photoproduct 16 can be formed by $6 \pi$ electrocyclization process followed by [4+2] cycloaddition of the intermediate 16' (Scheme 2).

Inserting a second styryl substituent at the $\beta$ position of the vinyl group of 1a (Figure 1), we prepared dimethoxy derivative $\mathbf{1 2}$ and obtained the system with extended conjugation, which can influence on the reaction course. Irradiation of compound $\mathbf{1 2}$ under the same conditions gave, after chromatograpfic separation on silica gel, very similar results (Scheme 2). Benzobicyclo[3.2.1]octadiene $\mathbf{1 7}$ and tricyclic derivative $\mathbf{1 8}$ were isolated. Their formation can be explained by the same mechanism as in the previous case of monomethoxy derivative 11. The difference in photochemical behaviour between mono- and dimethoxy-derivative is the prolonged time of irradiation to the full conversion in case of 12. This may be caused by insertion of the second styryl group, which might have influence on the additional stability of the starting molecule $\mathbf{1 2}$ in comparison to 11 . 


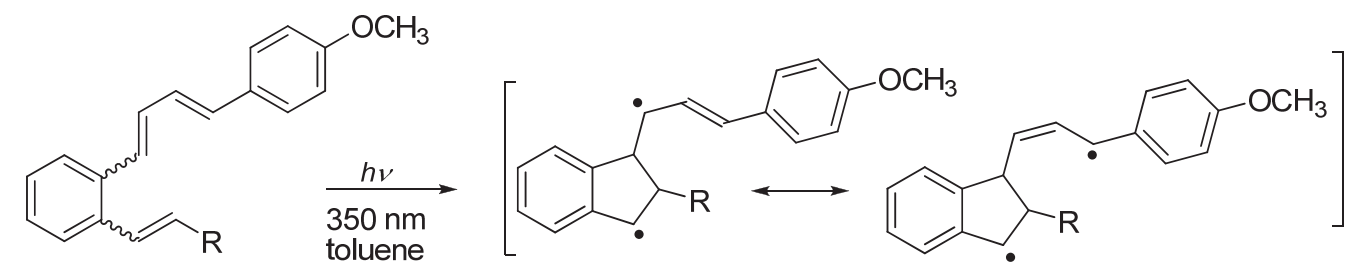<smiles>[R]C=Cc1ccccc1/C=C/C=C/c1ccc(OC)cc1</smiles><smiles>[Y5][I+3]</smiles>

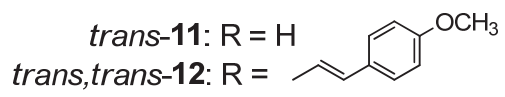
endo-15: $\mathrm{R}=\mathrm{H}(55 \%)$ trans,trans-12: $\mathrm{R}=$ 17: $\mathrm{R}=(24 \%)$<smiles>[R]C1C2C=CC(c3ccc(OC)cc3)C1C1C=CC=C2C1(C)C</smiles>
$h v, 350 \mathrm{~nm}$ toluene<smiles>[R]C1C=c2ccccc2=CC1/C=C/c1ccc(OC)cc1</smiles>

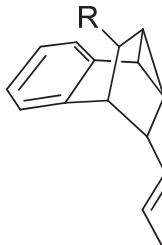<smiles>[124IH]</smiles><smiles>COc1ccccc1</smiles>
18': $\mathrm{R}=$

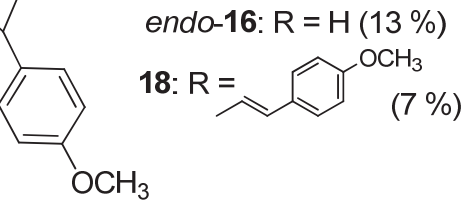

Scheme 2 .

Irradiation of starting compounds $\mathbf{1 3}$ and $\mathbf{1 4}$ gave different results. Under irradiation starting compounds $\mathbf{1 3}$ and $\mathbf{1 4}$ react giving new bicyclic cycloaddition products, interesting for further functionalization as it was shown for previously synthesized hetero-bicyclic photoproducts. ${ }^{21-23}$ After chromatographic purification of crude reaction mixture, only bicyclo derivatives 19 and 20 were isolated, respectively (Scheme 3.) The mecha-<smiles>[R]C=Cc1ccccc1/C=C\C=C\c1ccccc1</smiles>

13: $\mathrm{R}=\mathrm{CH}_{3}$ 14: $\mathrm{R}=\longrightarrow$<smiles>[R]C1Cc2ccccc2C1/C=C/c1ccccc1/C=C/C1c2ccccc2CC1/C=C\Cc1ccccc1</smiles>

19': $\mathrm{R}=\mathrm{CH}_{3}$ 20': $\mathrm{R}=\longrightarrow$

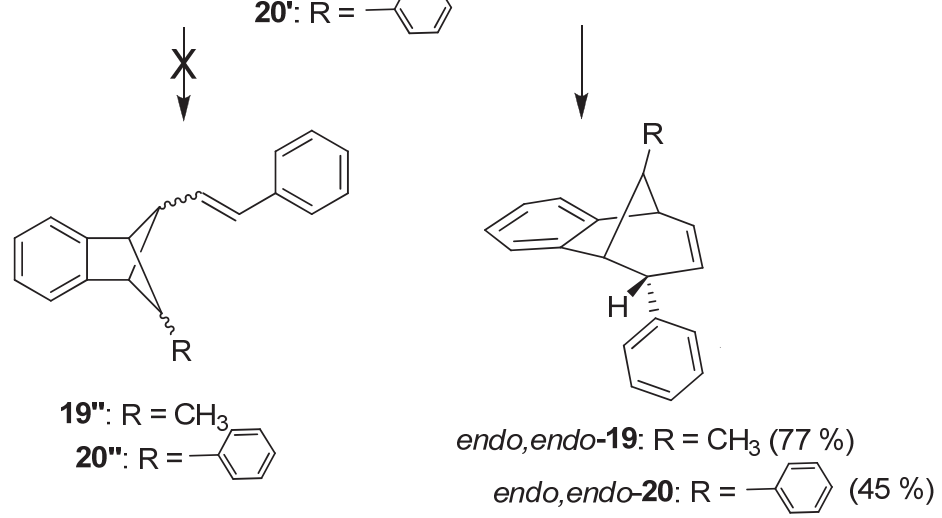

Scheme 3. 

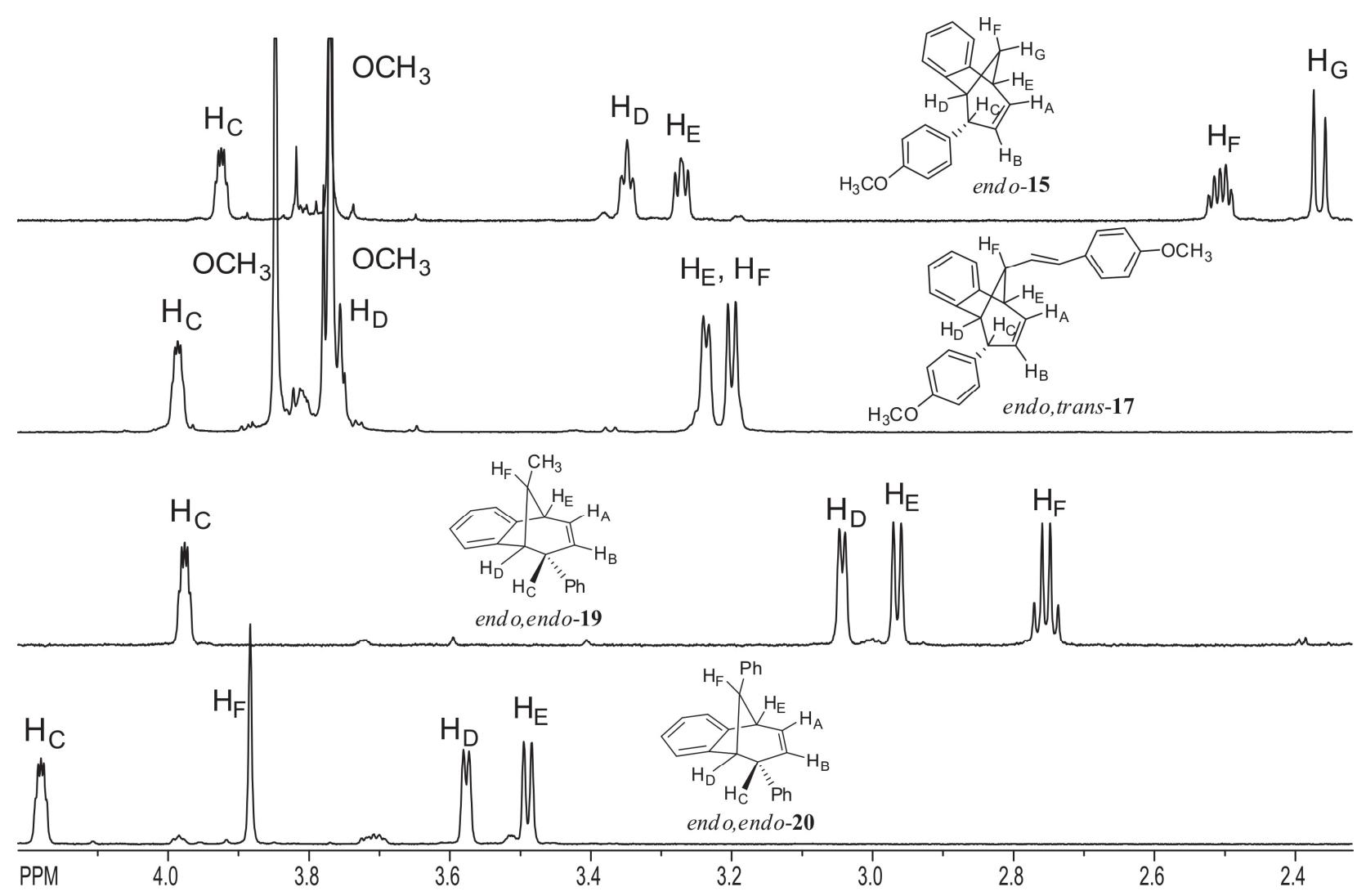

Figure 4. Part of ${ }^{1} \mathrm{H}$ NMR spectra of endo-15, endo,trans-17, endo, endo-19 and endo,endo-20 $\left(\mathrm{CDCl}_{3}, 600 \mathrm{MHz}\right)$.

nism of their formation, as seen previously, involves intramolecular [2+2] cycloaddition and 1,6 ring closure of intermediate 19' or 20'. Benzobicyclo[2.1.1] hexene derivative $\mathbf{2 1}$ or $\mathbf{2 2}$, which could be formed by 1,4-ring closure of 13 or $\mathbf{1 4}$, was not detected.

The structures of new photoproducts have been elucidated from their spectral data, ${ }^{1} \mathrm{H}$ NMR being most informative. Figure 4 shows comparison of aliphatic region of isolated bicyclic derivatives endo-15, endo,trans-17, endo,endo-19 and endo,endo-20. The recognizable pattern of ${ }^{1} \mathrm{H}$ NMR spectra of photoproducts in the region between 2.4 and $4.2 \mathrm{ppm}$ indicates the same bicyclo[3.2.1] octadiene structure. Depending on substitution at the $\beta$-position of the vinyl group, the aliphatic protons are shifted to higher or lower field. Protons of compounds endo,trans-17 and endo,endo-19, which possess the second phenyl group are deshielded and shifted to lower field in comparison to protons of endo-15 and endo,endo-20. The accent is on $\mathrm{H}_{\mathrm{F}}$ proton, which is shifted from $2.5 \mathrm{ppm}$ (in endo-15) to $3.2 \mathrm{ppm}$ (endo,trans-17) and from $2.8 \mathrm{ppm}$ (endo,endo-19) to 3.9 ppm (endo,endo-20). Tricyclic derivatives endo-16 and endo,trans-18 has a completely different pattern in ${ }^{1} \mathrm{H}$ NMR spectrum (Figure 5) compared to bicyclic photoproducts. Patterns of aliphatic protons of both derivatives are very similar. The difference is disappearance of $\mathrm{H}_{\mathrm{D}}$ proton in endo,trans-18 due to the entry of the second styryl or benzyl group to the molecule. For the same reason proton $\mathrm{H}_{\mathrm{G}}$ is strongly shifted to lower field from $1.2 \mathrm{ppm}$ in endo-16 to $3.1 \mathrm{ppm}$ in endo,trans-18.

Methoxy group might have electronic and/or steric effects on the photochemical behaviour of the analyzed compound. But the results obtained under irradiation of mono-methoxy derivative $\mathbf{1 1}$ in comparison with previously reported unsubstituted $\mathbf{1 a}$ and chloro-derivative 1d are very similar. In all cases benzobicyclo[3.2.1]octadiene structures endo-3, endo-8 and endo15 are isolated, respectively, as main product. Besides, methoxy derivative $\mathbf{1 1}$ gives tricyclic derivative endo16. The yield on the isolated bicyclic derivative is the largest by use of the unsubstituted compound (90\%) where the steric and electronic effects are reduced. In case of chloro derivative the yield of the isolated product $(77 \%)$ is slightly higher compared to the methoxy compound endo-15 (55\%), having potentially increased steric and electronic influence relative to a chlorine atom. Electronic effects may have influence on the reaction course, as in the case of methoxy derivative the reaction was less selective giving two photoproducts, in contrary to the previously described derivatives, where only the bicyclo derivative is isolated. In case of dibutadiene derivatives, unsubstituted compound 2a under 


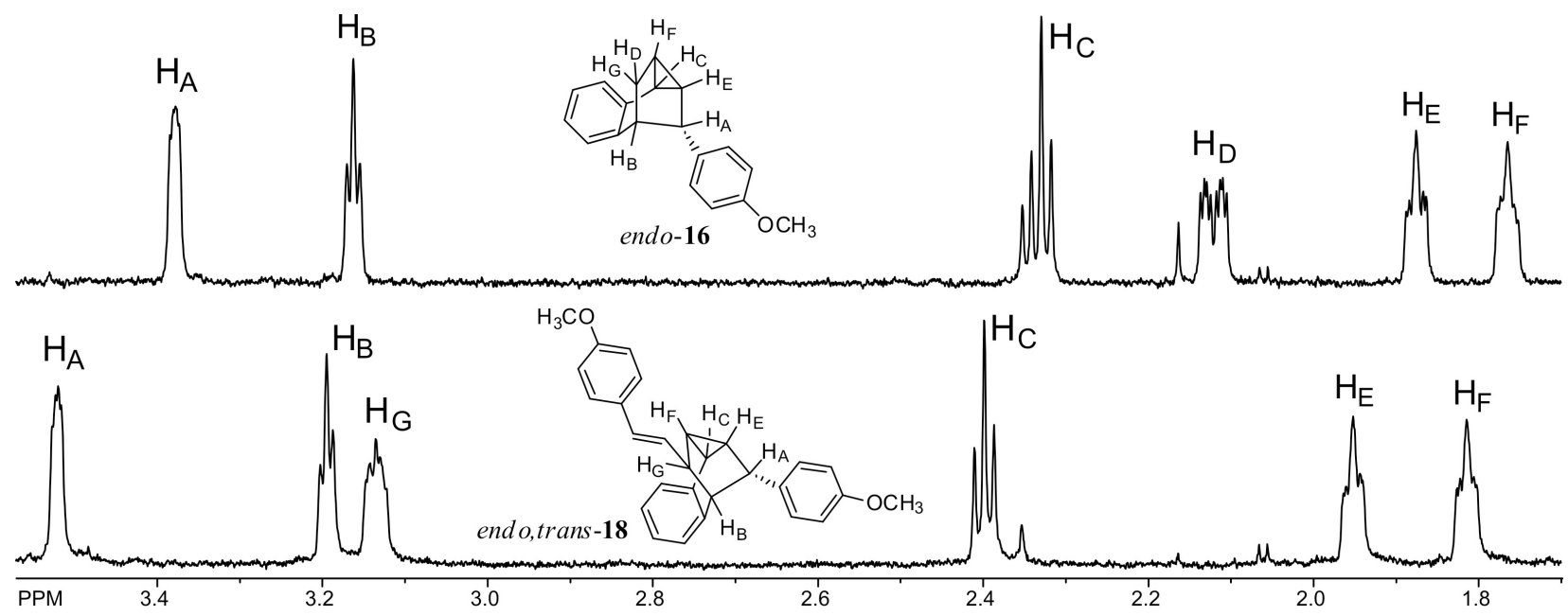

Figure 5. Part of ${ }^{1} \mathrm{H}$ NMR spectra of endo-16 and endo,trans-18 $\left(\mathrm{CDCl}_{3}, 600 \mathrm{MHz}\right)$.

irradiation gives only benzobicyclo[3.2.1] octadiene structure (endo,trans-4). Chloro and methoxy derivatives, due to their different steric and electronic effect give the same type of products, benzobicyclo[3.2.1] octadiene structure (endo,trans-9, endo,trans17) and tricyclic derivative (endo-10, endo,trans-18). On the other hand compounds $\mathbf{1 3}$ and $\mathbf{1 4}$ under irradiation behave as the unsubstituted derivative 1a giving selectively only bicyclic structures endo,endo-19 and endo,endo-20, respectively, but in lower yields.

In cases of all bicyclic photoproducts the ring closure predominantly gives endo-isomers. The stereoselectivity of the cycloaddition reaction and preferable ring closure to endo-isomer can be ascribed to the stabilization of the transition state in endo-orientation by the strong attractive intramolecular $\pi-\pi$ interactions of the benzo-phenyl. ${ }^{30-32}$

\section{CONCLUSION}

Different substituted butadiene derivatives under irradiation show not so diverse mechanism of photochemical behavior but with different selectivity and yields. Considering the nature and the position of the substituents, new polycyclic structures are formed. Starting materials 13 and 14 under irradiation give stereospecific reaction to benzobicyclo[3.2.1] octadiene structure of endo,endo19 and endo,endo-20, respectively. On the other hand, methoxy derivatives $\mathbf{1 1}$ and $\mathbf{1 2}$ are less selective. Besides [2+2] cycloaddition to benzobicyclo[3.2.1]octadiene structures endo-15 and endo,trans-17, they react in photoinduced [4+2] cycloaddition giving tricyclic derivatives (endo-16 and endo,trans-18) as minor products. Understanding the effects of substituents is very important in the field of preparative photochemistry and predicting the mechanism and yields. The slightly change on the structure of starting materials affected the selectivity, stereoselectivity and the reaction course leading to bicyclic photoproducts in moderate to good yields (24-77\%). All new prepared and fully characterized bicyclic molecules are suitable substrates for further efficient photocatalytic oxygenation reactions in course of getting new functionalized polycycles, potentially biologically more active compounds with greater similarity to the structure of some naturally occurred terpenes.

Supplementary Materials. - Supporting informations to the paper are enclosed to the electronic version of the article. These data can be found on the website of Croatica Chemica Acta (http://public.carnet.hr/ccacaa).

Acknowledgements. This research was founded by grants from the Croatian Ministry of Science, Education and Sports (1250982933-2926 and 098-0982929-2917) and University of Zagreb short term scientific support under the title "Functionalization of the benzobicyclo[3.2.1]octadiene skeleton using photocatalytic oxygenation reactions".

\section{REFERENCES}

1. I. Škorić, M. Šmehil, Ž. Marinić, K. Molčanov, B. Kojić-Prodić, and M. Šindler-Kulyk, J. Photochem. Photobiol. A: Chemistry 207 (2009) 190.

2. I. Škorić, I. Kikaš, M. Kovács, L. Fodor, Ž. Marinić, K. Molčanov, B. Kojić-Prodić, and O. Horváth, J. Org. Chem. 76 (2011) 8641

3. D. Vuk, D. Potroško, M. Šindler-Kulyk, Ž. Marinić, K. Molčanov, B. Kojić-Prodić, and I. Škorić, J. Mol. Struct. 1051 (2013) 1.

4. R. C. Hahn and L. J. Rothman, J. Am. Chem. Soc. 91 (1969) 2409.

5. Z. Goldschmidt and U. Gutman, Tetrahedron 30 (1974) 3327.

6. R. P. Johnson, A. Exarchou, Ch. W. Jeffrd, and R. C. Hahn, J. Org. Chem. 42 (1977) 3758.

7. D. Wege, J. Org. Chem. 55 (1990) 1667.

8. S. Yamamura, Y. Shizuri, H. Shigemori, Y. Okuno, and M. Ohkubo, Tetrahedron 47 (1991) 635. 
9. L. N. Mander, Chem. Rev. 92 (1992) 573.

10. T. A. Engler, D. D. Wie, and M. A. Letavic, Tetrahedron Lett. 34 (1993) 1429

11. H. Nakajima, K. Isomi, T. Hamasaki, and M. Ichinoe, Tetrahedron Lett. 35 (1994) 9597.

12. H. Lin and S. J. Danishefsky, Angew. Chem. Int. Ed. 42 (2003) 36.

13. M. H. Rosen, I. Fengler, and G. Bonet, J. Med. Chem. 19 (1976) 414.

14. H. Nagata, M. Kawamura, and K. Ogasawara, Synthesis $\mathbf{1 3}$ (2000) 1825 .

15. M.-H. Filippini, R. Faure, and J. Rodriguez, J. Org. Chem. 60 (1995) 6872

16. M.-H. Filippini and J. Rodriguez, Chem. Rev. 99 (1999) 27.

17. M. Iwashima, I. Terada, K. Okamoto, and K. Iguchi, J. Org. Chem. 67 (2002) 2977.

18. T. Ito, T. Tanaka, Z. Ali, Y. Akao, Y. Nozawa, Y. Takahashi, R. Sawa, K. Nakaya, J. Murata, D. Darnaedi, and M. Iinuma, Heterocycles 63 (2004) 129.

19. T. Ito, T. Tanaka, M. Iinuma, I. Iliya, K. Nakaya, Z. Ali, Y. Takahashi, R. Sawa, Y. Shirataki, J. Murata, and D. Darnaedi, Tetrahedron $\mathbf{5 9}$ (2003) 5347.

20. P. S. Jones, P. W. Smith, G. W. Hardy, P. D. Howes, R. J. Upton, and R. C. Bethell, Bioorg. Med. Chem. Lett. 9 (1999) 605.

21. I. Kikaš, O. Horváth, and I. Škorić, Tetrahedron Lett. 52 (2011)
6255.

22. I. Kikaš, O. Horváth, and I. Škorić, J. Mol. Struct. 1034 (2013) 62.

23. D. Vuk, I. Kikaš, K. Molčanov, O. Horváth, and I. Škorić, $J$. Mol. Struct. 1063 (2014) 83.

24. H. Hennig, J. Behling, R. Meusinger, and L. Weber, Chem. Ber. 128 (1995) 229.

25. H. Hennig, Coord. Chem. Rev. 182 (1999) 101 and references therein.

26. A. Maldotti, L. Andreotti, A. Molinari, G. Varani, G. Cerichelli, and M. Chiarini, Green Chem. 3 (2001) 42.

27. M. Hajimohammadi, F. Bahadoran, S. S. H. Davarani, and N. Safari, Reac. Kinet. Mech. Cat. 99 (2010) 243.

28. D. Vuk, K. Molčanov, and I. Škorić, J. Mol. Struct. 1065-1066 (2014) 43.

29. D. Vuk, K. Molčanov, and I. Škorić, J. Mol. Struct. 1068 (2014) 124.

30. C. A. Hunter and K. M. Sanders, J. Am. Chem. Soc. 112 (1990) 5525 .

31. J. Nishimura, Y. Nakamura, Y. Hayashida, and T. Cudo, Acc. Chem. Res. 33 (2000) 679.

32. (a) E. A. Meyer, R. K. Castellano, and F. Dietrich, Angew. Chem. 115 (2003) 1244; (b) E. A. Meyer, R. K. Castellano, and F. Dietrich, Angew. Chem. Int. Ed. 42 (2003) 1210. 


\title{
Synthesis of new polycyclic structures by photoirradiation of butadiene derivatives
}

\author{
Dragana Vuk ${ }^{\mathrm{a}}$, Željko Marinić ${ }^{\mathrm{b}}$, Irena Škorić ${ }^{\mathrm{a}, 1}$ \\ ${ }^{a}$ Department of Organic Chemistry, Faculty of Chemical Engineering and Technology, \\ University of Zagreb, Marulićev trg 19, 10000 Zagreb, Croatia; \\ ${ }^{b}$ Center for NMR, Rudjer Bošković Institute, Bijenička cesta 54, 10000 Zagreb, Croatia;
}

${ }^{1} \mathrm{H}$ NMR and UV spectrum for compound endo-15 2

${ }^{1} \mathrm{H}$ NMR, ${ }^{13} \mathrm{C}$ NMR, UV and IR spectrum for compound endo-16

${ }^{1} \mathrm{H}$ NMR, UV and IR spectrum for compound endo,trans-17

${ }^{1} \mathrm{H}$ NMR, UV and IR spectrum for compound endo,trans-18

${ }^{1} \mathrm{H}$ NMR and IR spectrum for compound endo,endo-19

${ }^{1} \mathrm{H}$ NMR, ${ }^{13} \mathrm{C}$ NMR, NOESY and IR spectrum for compound endo,endo-20

${ }^{1}$ E-mail: iskoric@fkit.hr 


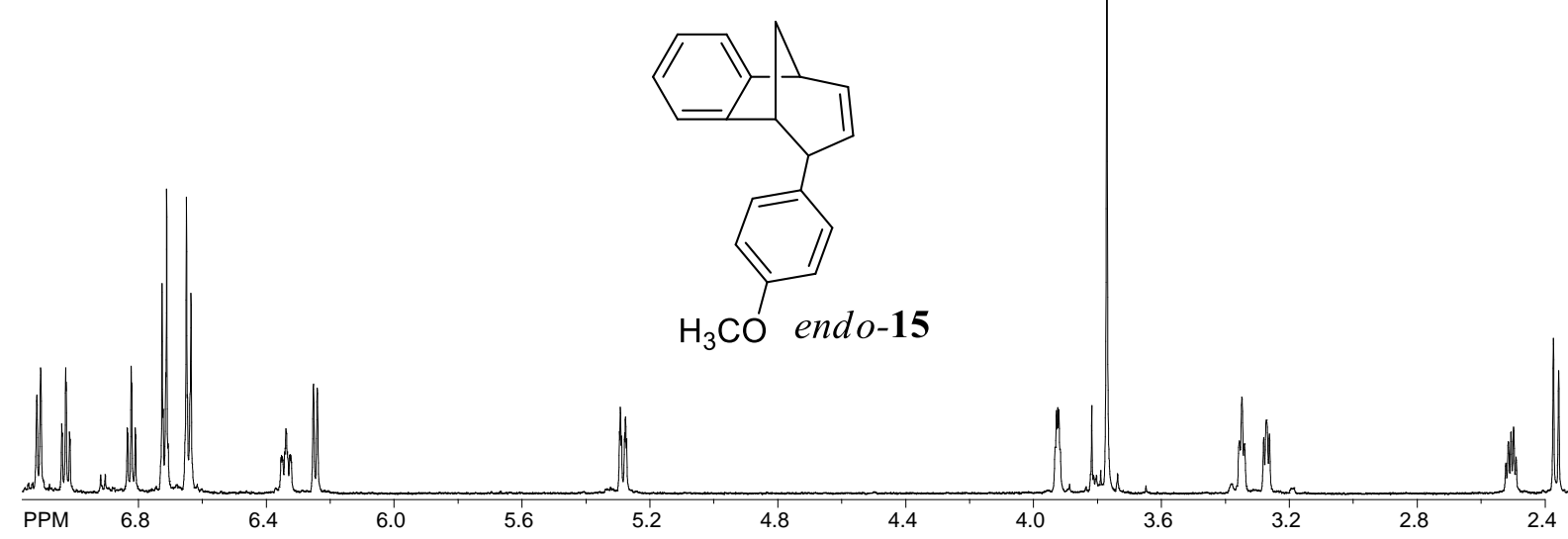

${ }^{1} \mathrm{H}$ NMR spectrum $\left(\mathrm{CDCl}_{3}, 600 \mathrm{MHz}\right)$ for compound endo-15

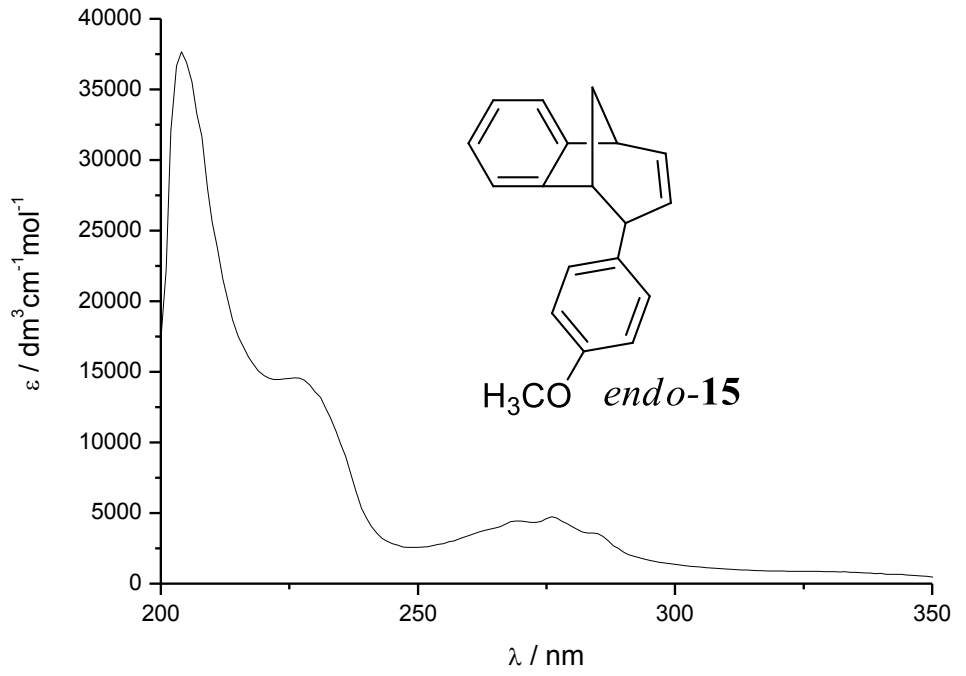

UV (96\% ethanol) spectrum for compound endo-15 


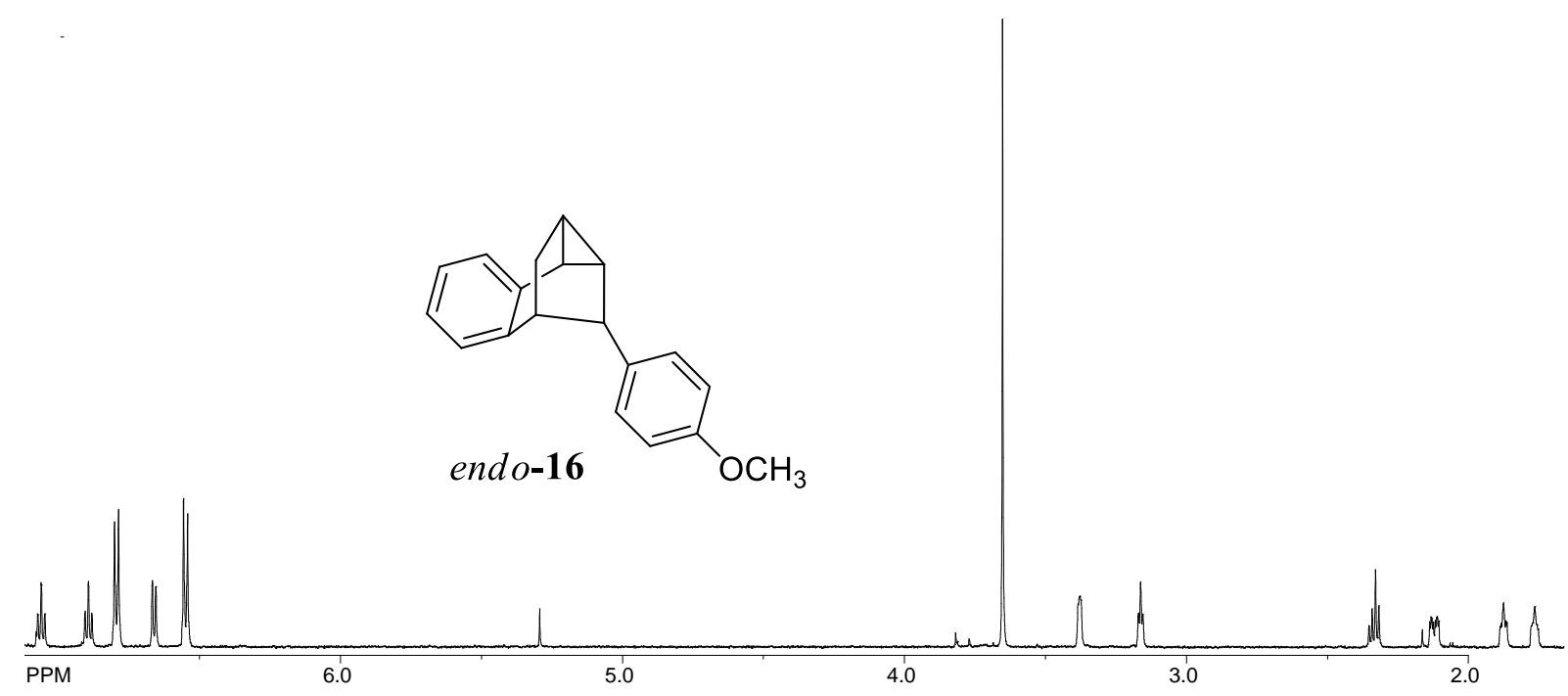

${ }^{1} \mathrm{H} \mathrm{NMR}$ spectrum $\left(\mathrm{CDCl}_{3}, 600 \mathrm{MHz}\right)$ for compound endo-16

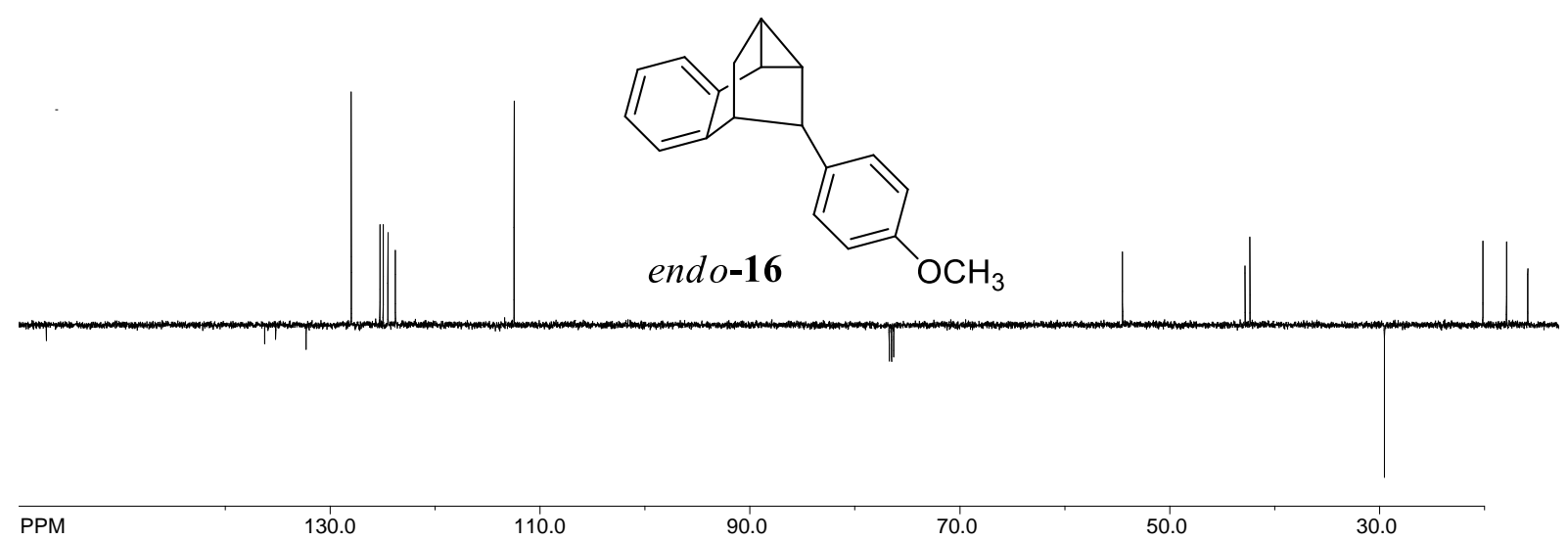

${ }^{13} \mathrm{C}$ NMR spectrum $\left(\mathrm{CDCl}_{3}, 150 \mathrm{MHz}\right)$ for compound endo-16 


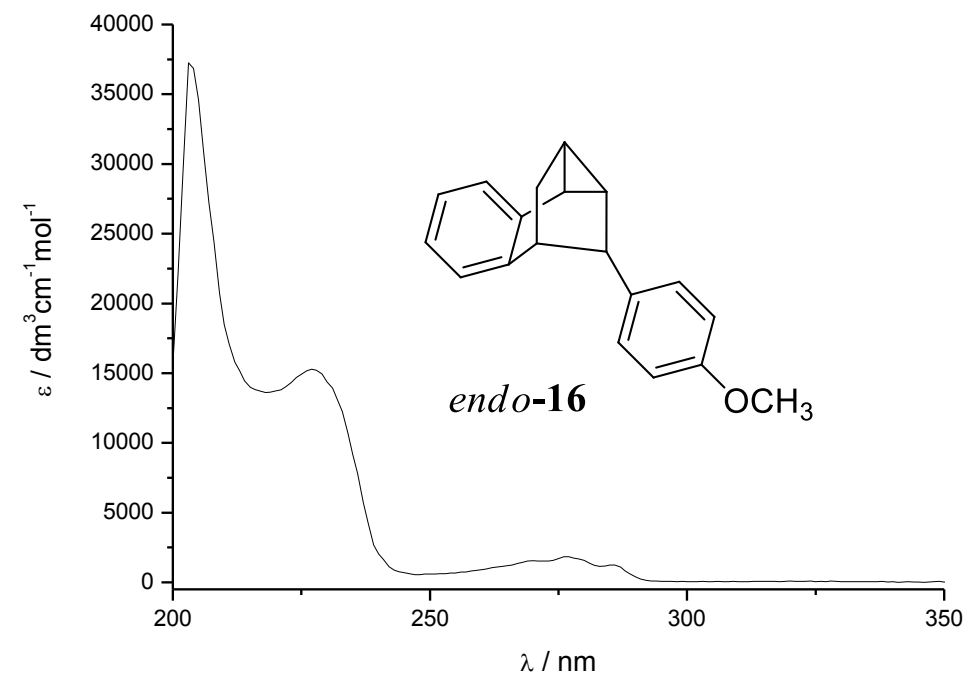

UV (96\% ethanol) spectrum for compound endo-16

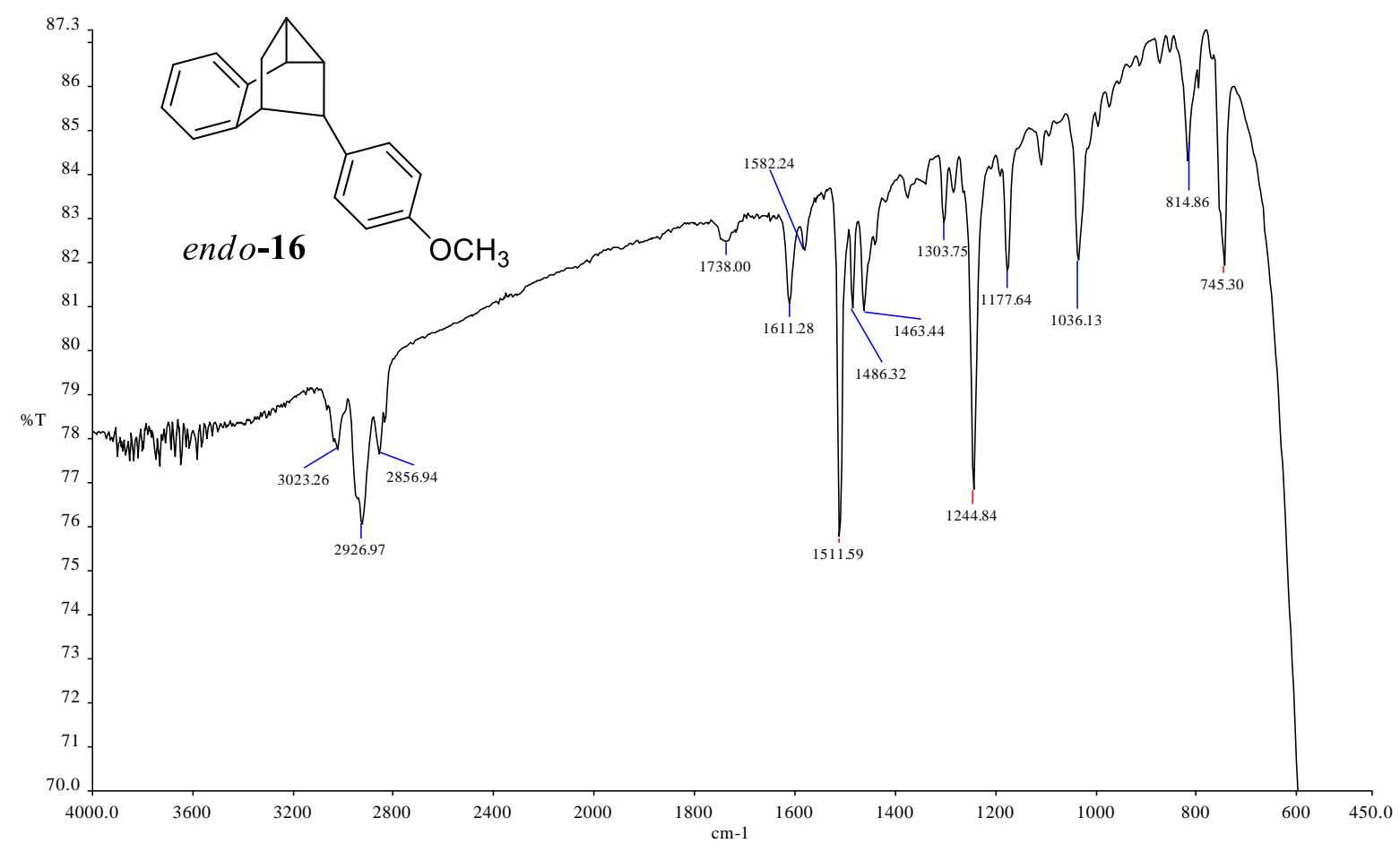

IR (evaporated film from $\mathrm{CH}_{2} \mathrm{Cl}_{2}$ ) spectrum for compound endo-16 


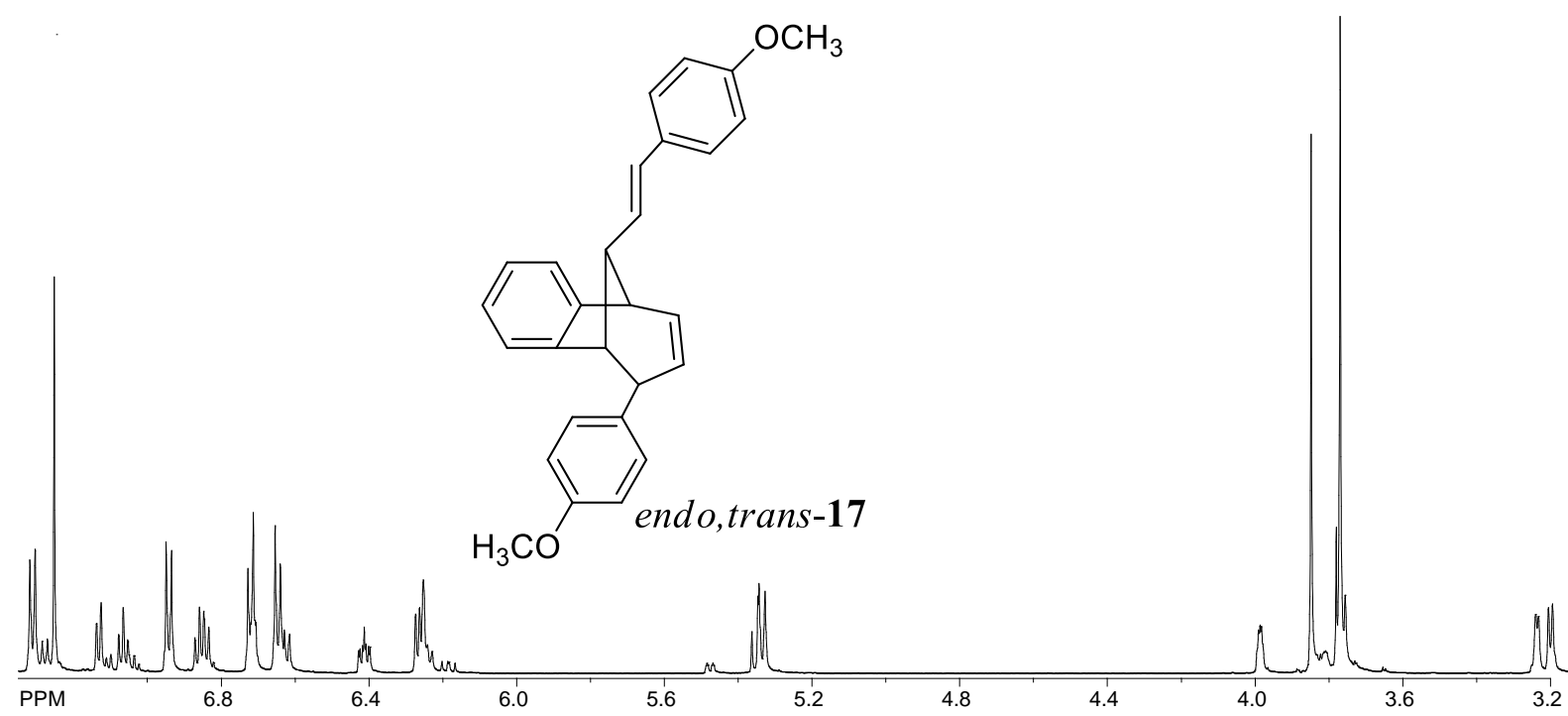

${ }^{1} \mathrm{H} \mathrm{NMR}$ spectrum $\left(\mathrm{CDCl}_{3}, 600 \mathrm{MHz}\right)$ for compound endo,trans-17

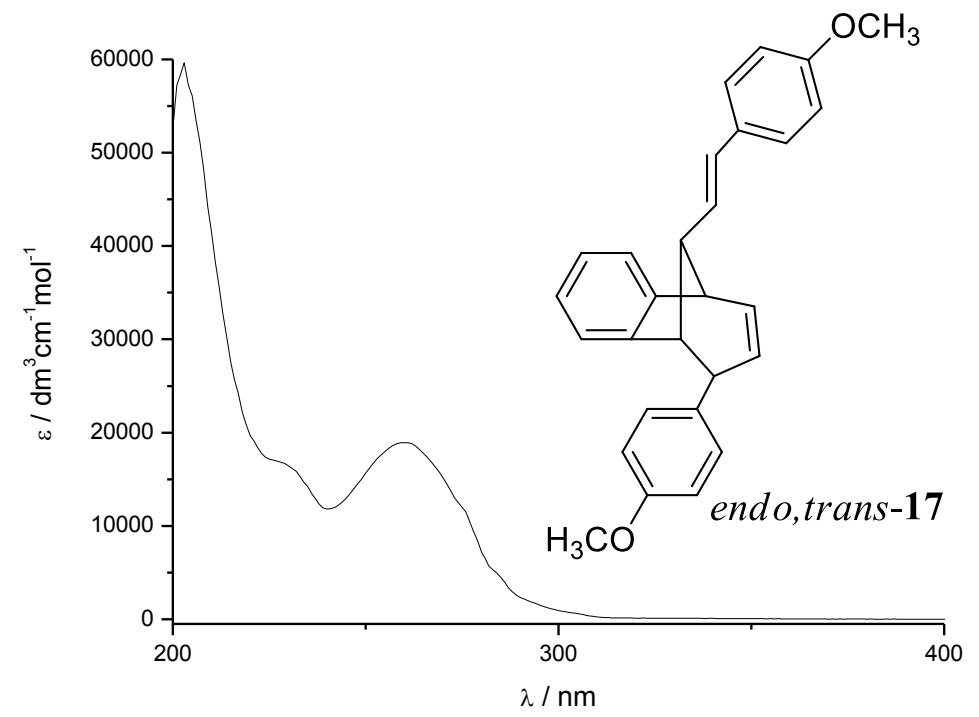

UV (96\% ethanol) spectrum for compound endo,trans-17 


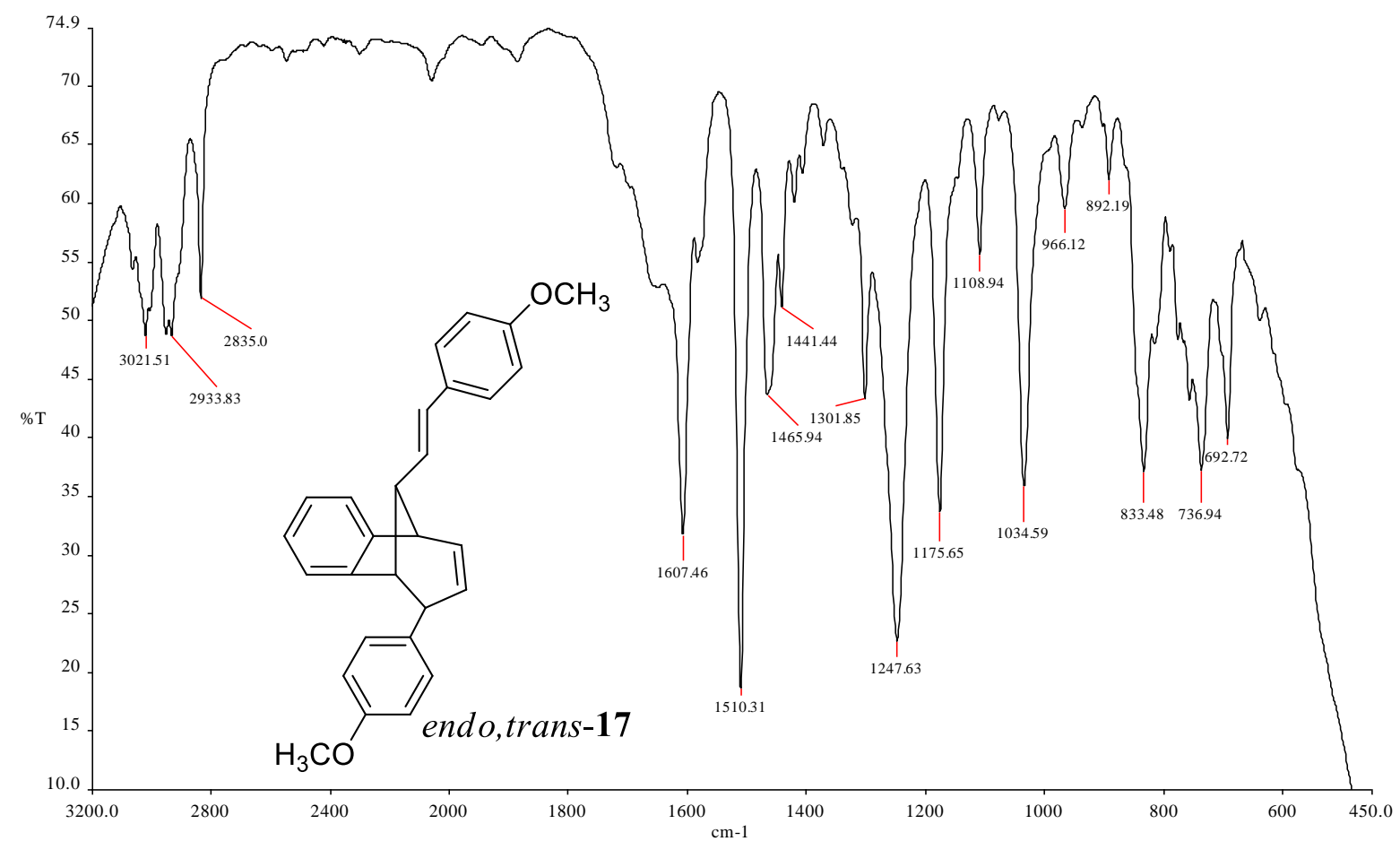

IR (evaporated film from $\mathrm{CH}_{2} \mathrm{Cl}_{2}$ ) spectrum for compound endo,trans-17

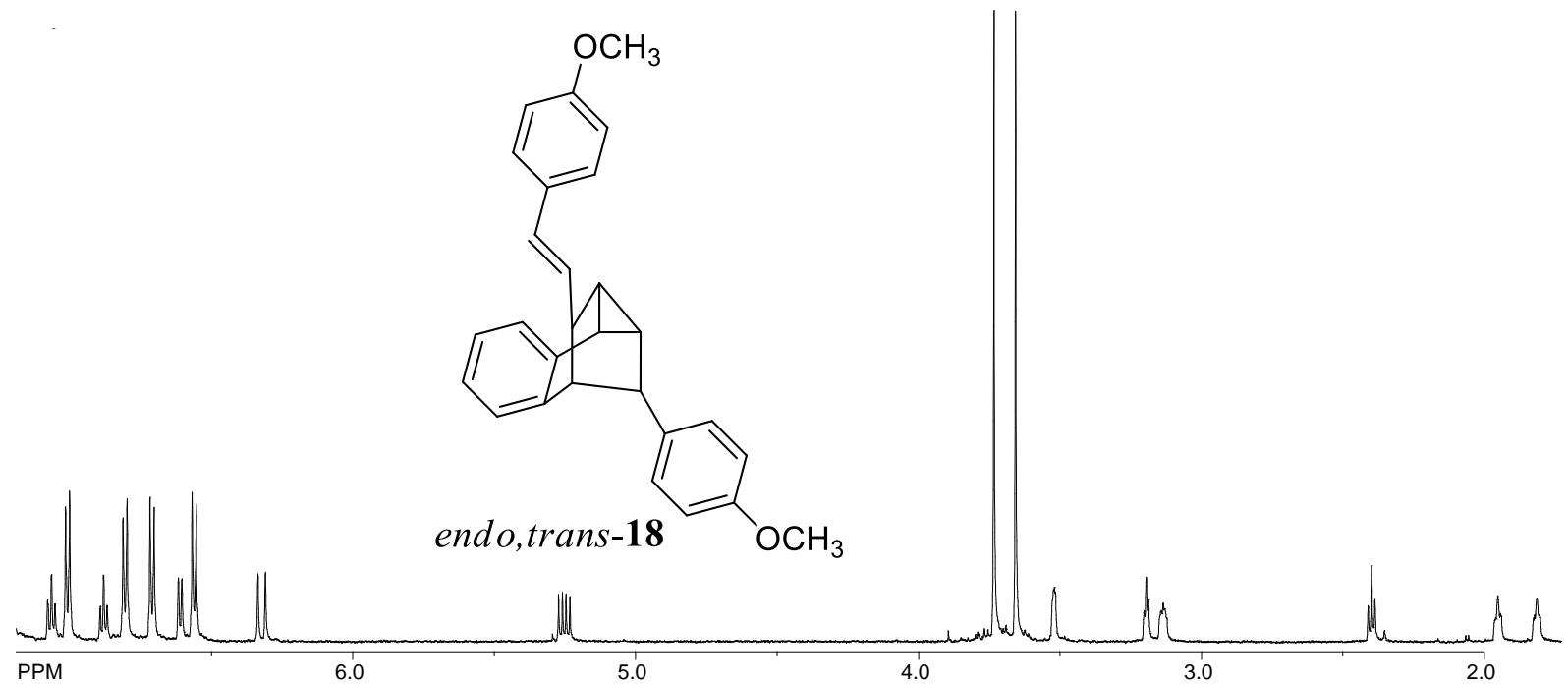

${ }^{1} \mathrm{H}$ NMR spectrum $\left(\mathrm{CDCl}_{3}, 600 \mathrm{MHz}\right)$ for compound endo,trans-18 


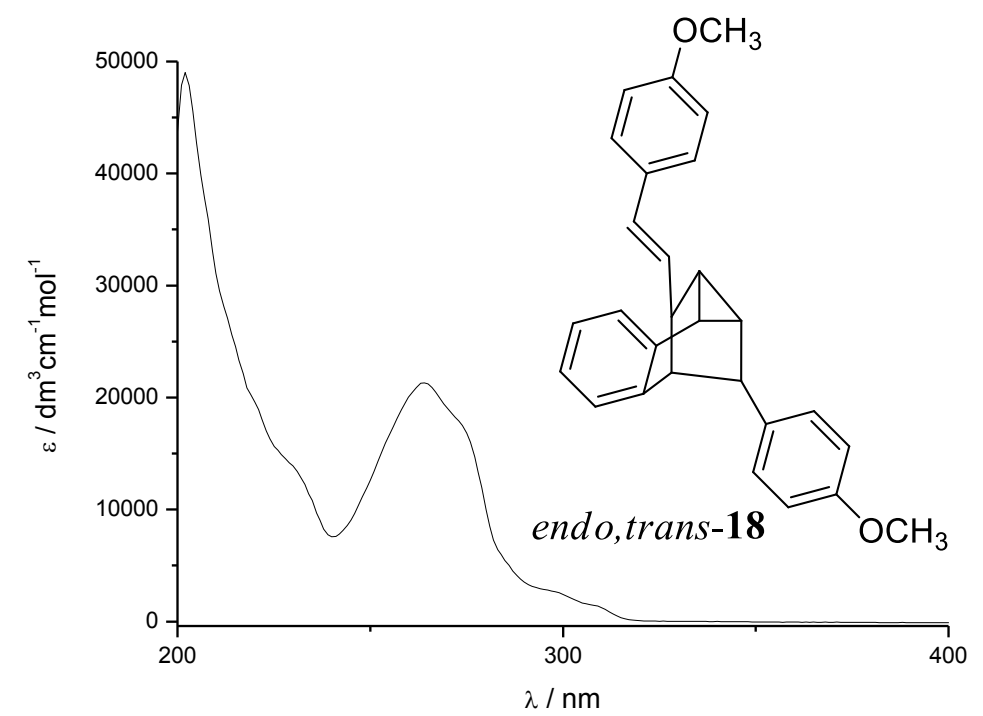

UV (96\% ethanol) spectrum for compound endo,trans-18

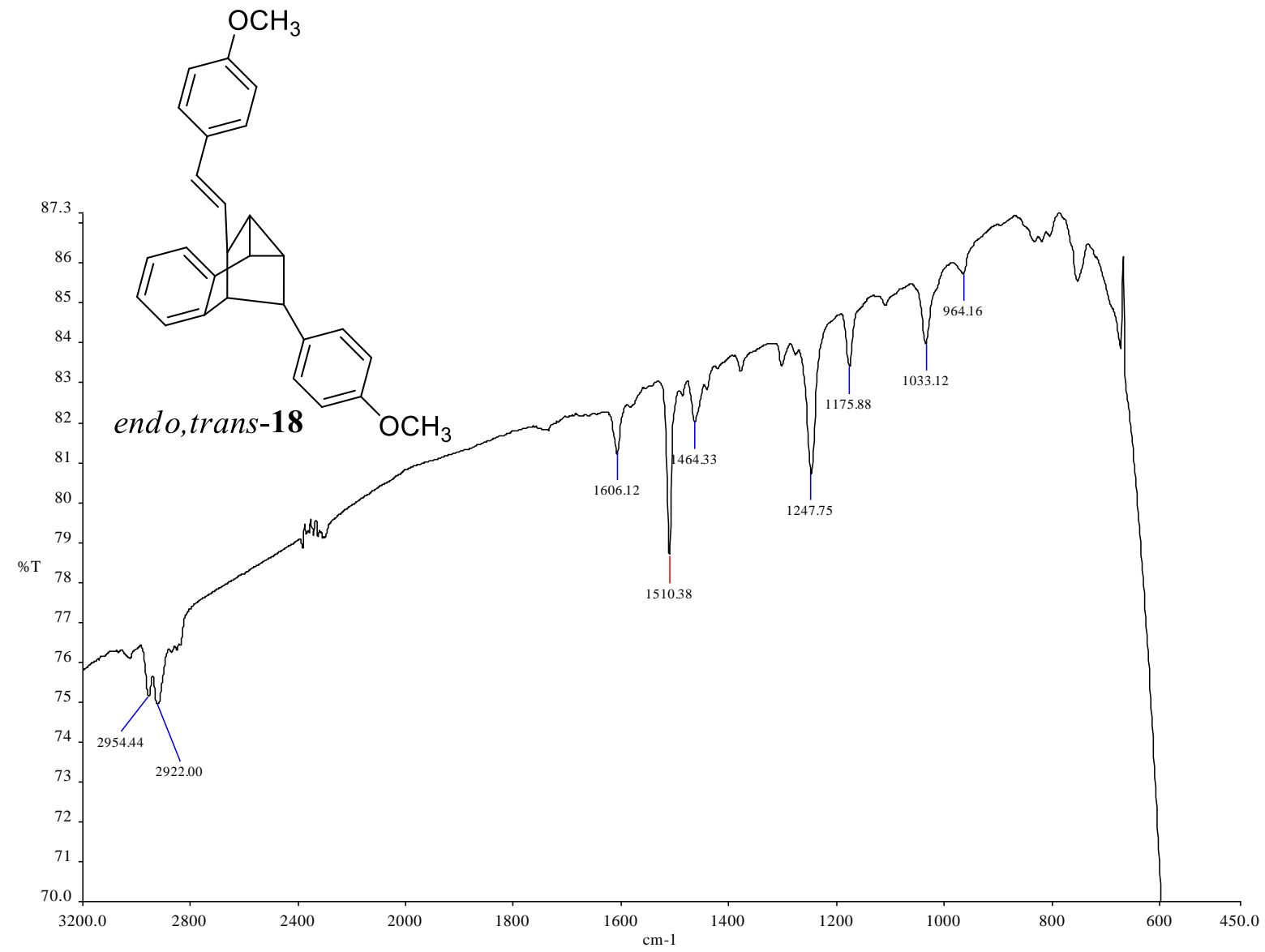

IR (evaporated film from $\mathrm{CH}_{2} \mathrm{Cl}_{2}$ ) spectrum for compound endo,trans-18 


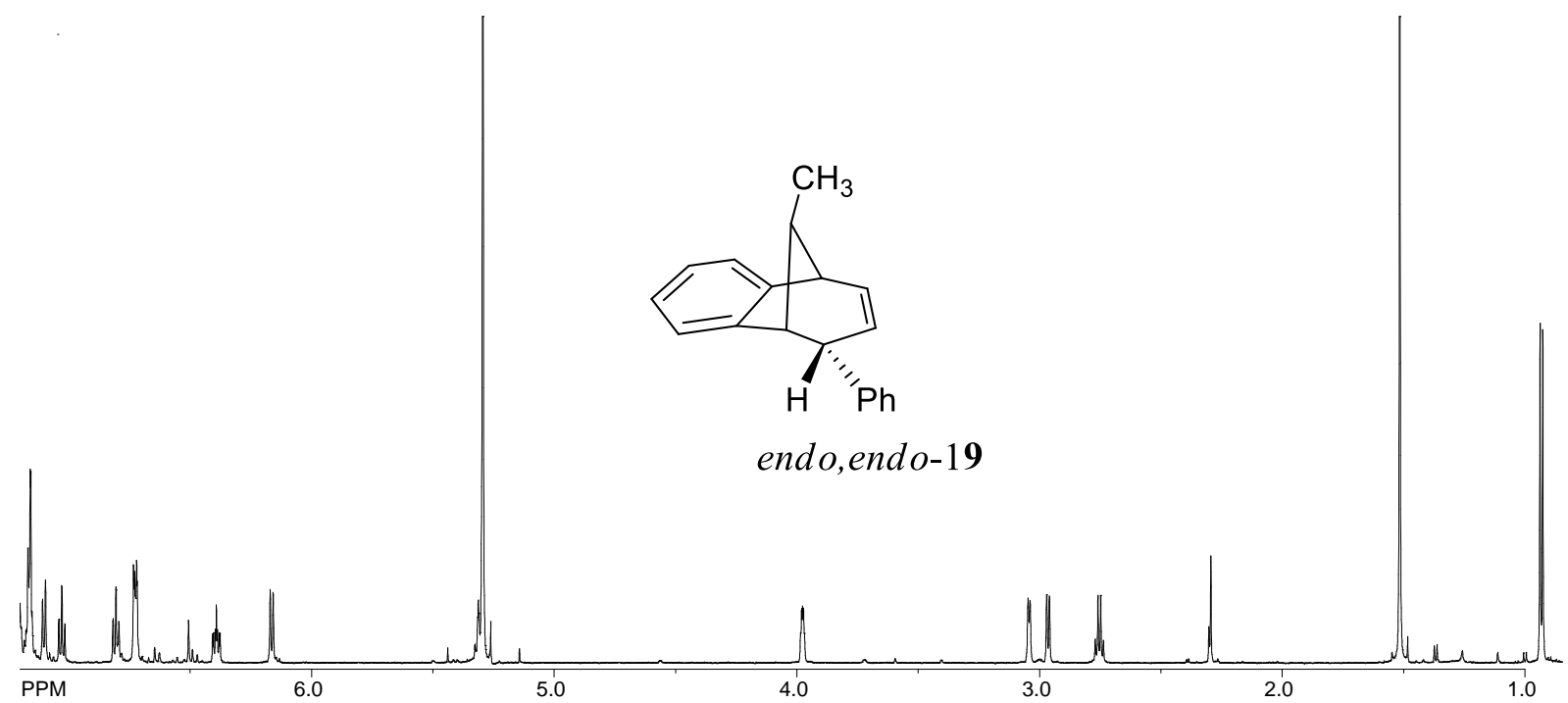

${ }^{1} \mathrm{H}$ NMR spectrum $\left(\mathrm{CDCl}_{3}, 600 \mathrm{MHz}\right)$ for compound endo,endo-19

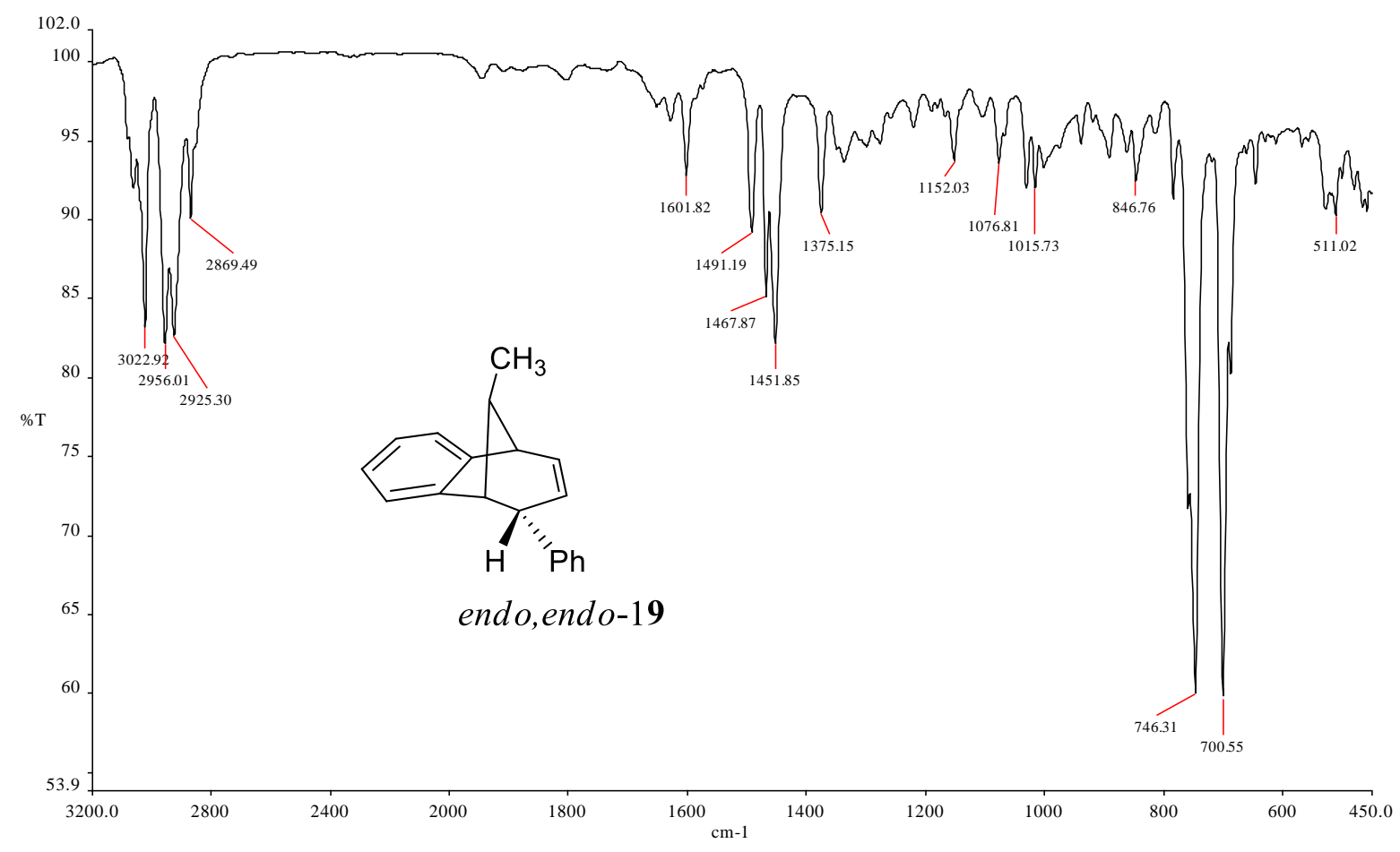

IR (evaporated film from $\mathrm{CH}_{2} \mathrm{Cl}_{2}$ ) spectrum for compound endo,endo-19 


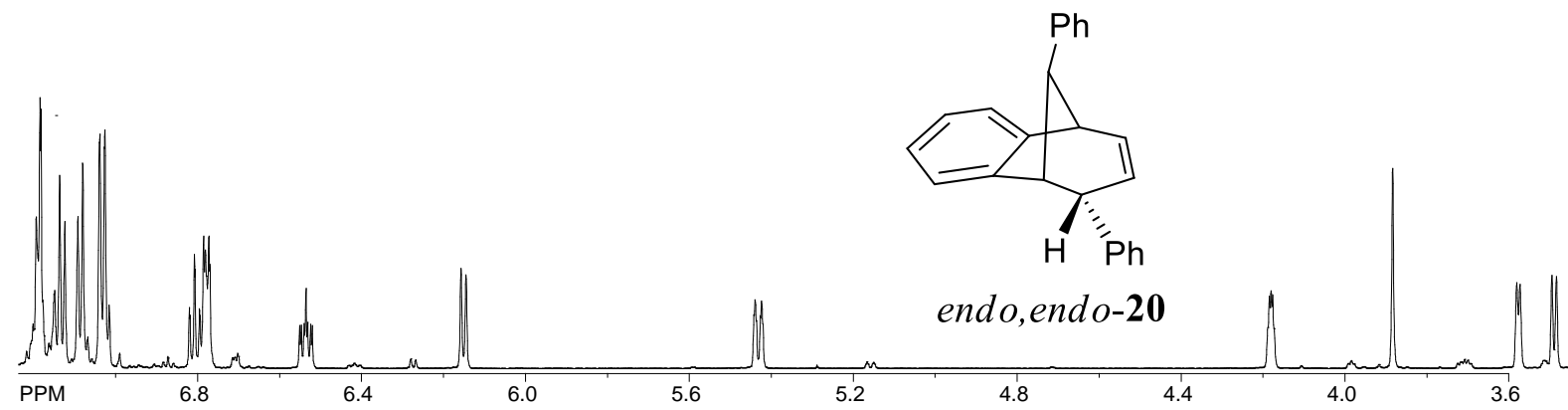

${ }^{1} \mathrm{H}$ NMR spectrum $\left(\mathrm{CDCl}_{3}, 600 \mathrm{MHz}\right)$ for compound endo,endo-20

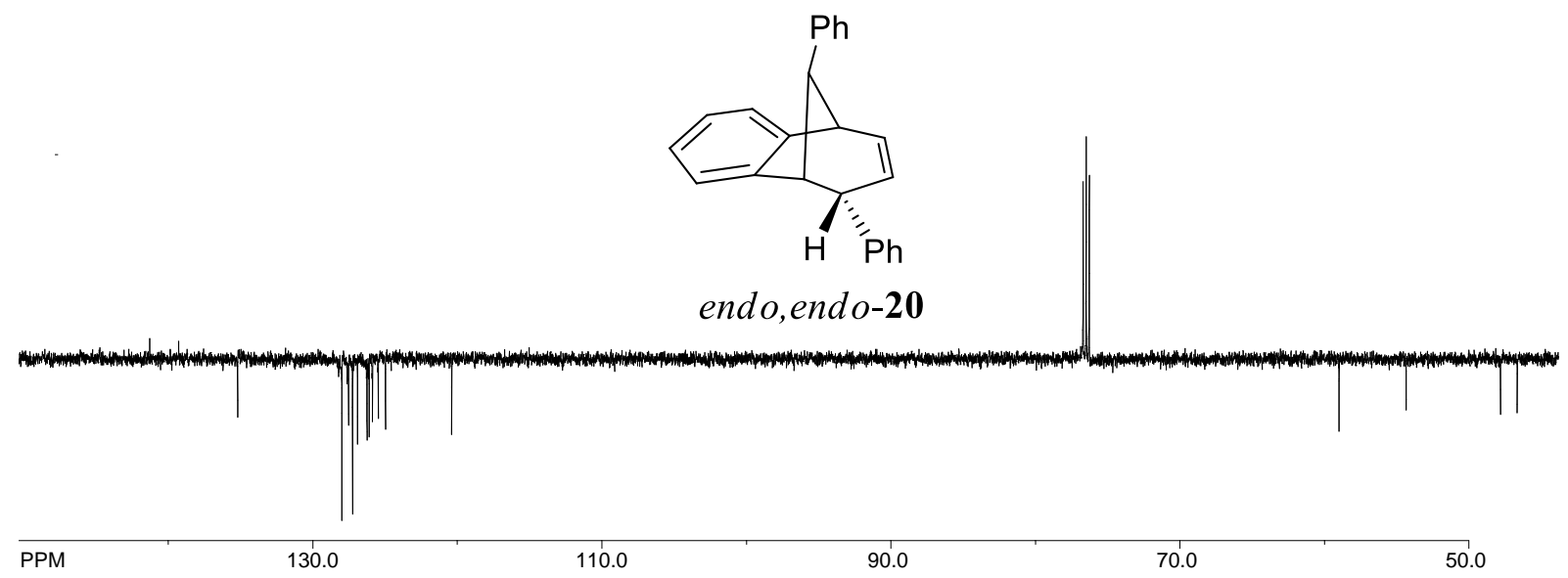

${ }^{13} \mathrm{C}$ NMR spectrum $\left(\mathrm{CDCl}_{3}, 150 \mathrm{MHz}\right)$ for compound endo,endo-20 


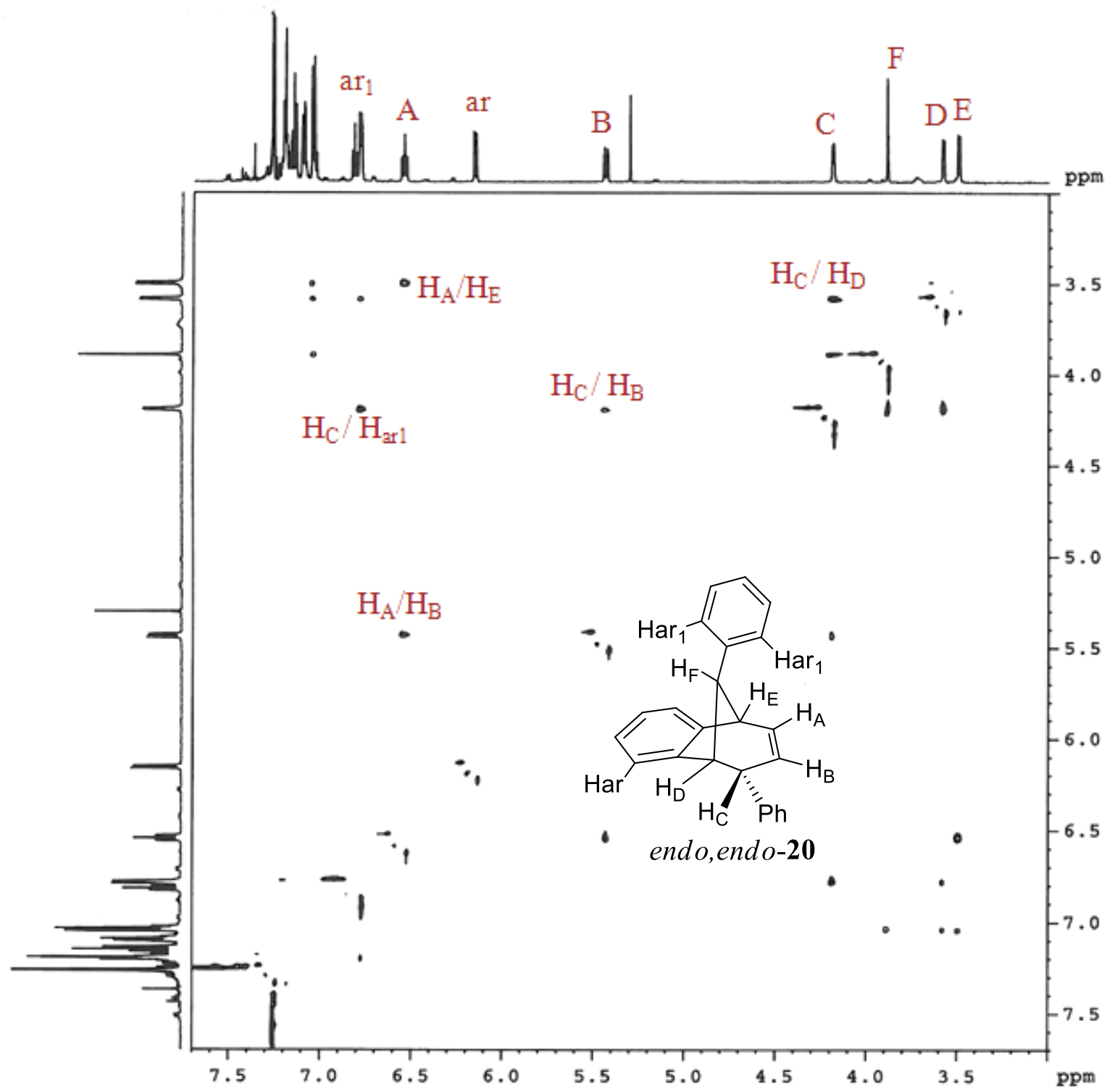

NOESY spectrum $\left(\mathrm{CDCl}_{3}\right)$ for compound endo, endo-20 


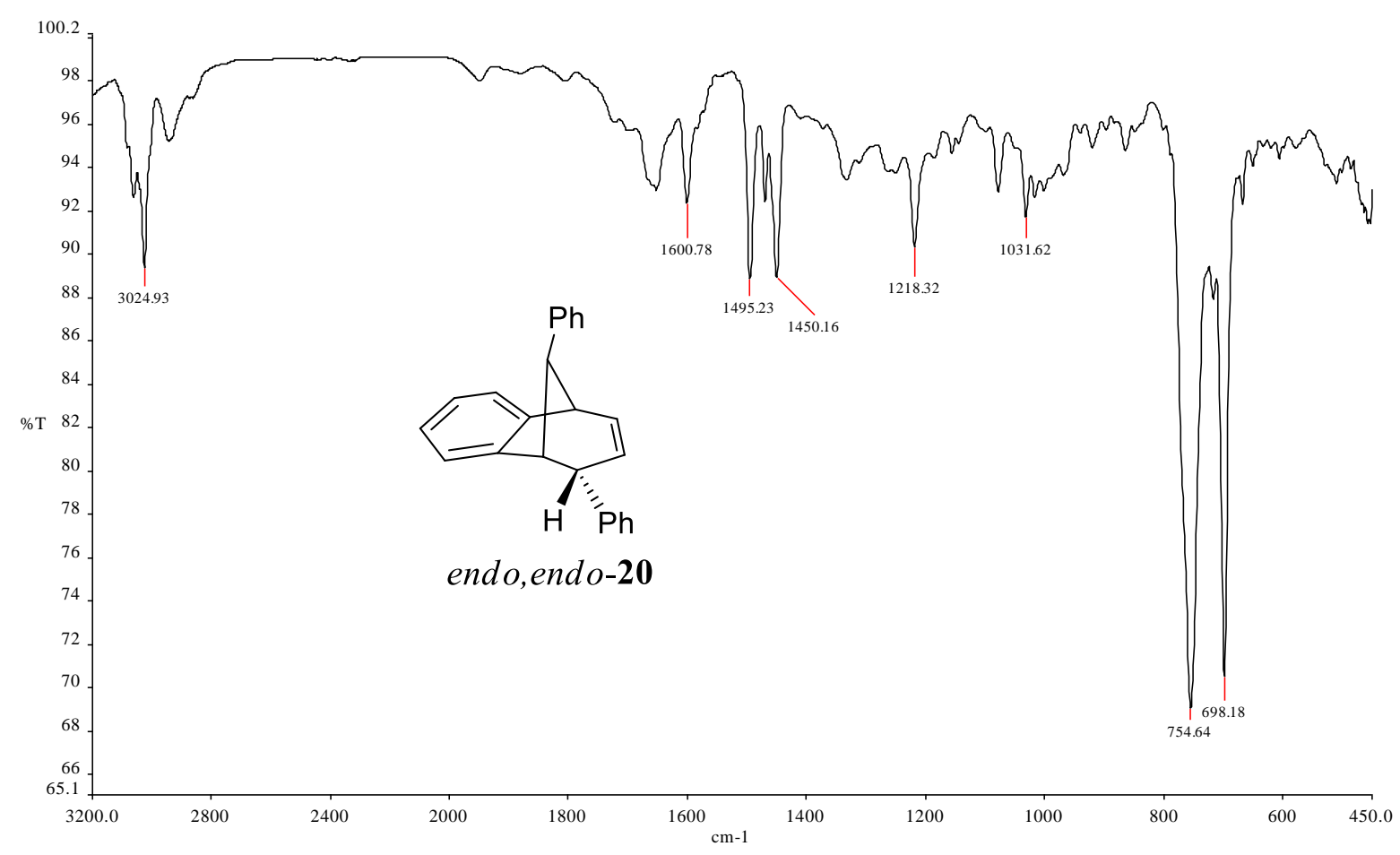

IR (evaporated film from $\mathrm{CH}_{2} \mathrm{Cl}_{2}$ ) spectrum for compound endo,endo-20 